\title{
Spectroscopic and DFT Studies of the Molecular Geometry and Electronic Structure of Classical and Non-classical Radical Ions derived from 7-Benzhydrylidenenorbornene Analogues
}

\author{
Hayato Namai, Hiroshi Ikeda, Takashi Hirano, Hideki Ishii, and Kazuhiko Mizuno
}

(1) General Method. Melting points were obtained with a Yamato MP-21 apparatus, and were uncorrected. Elemental analyses were performed by the Research and Analytical Center for Giant Molecules, Graduate School of Science, Tohoku University. IR spectra were measured with a JASCO IR-800 spectrometer. Low- and high-resolution electron ionization mass spectra (MS) were recorded with a Hitachi M80-B mass spectrometer. Fast atom bombardment mass spectra (FABMS) were recorded on a ThermoQuest TSQ 700 instrument using a Xe atom beam for ionization and $m$-Nitrobenzylalcohol ( $m$-NBA) as a matrix. ${ }^{1} \mathrm{H}$ and ${ }^{13} \mathrm{C}$ NMR spectra were recorded on a JEOL GX-270 instrument $\left(270 \mathrm{MHz}\right.$ for ${ }^{1} \mathrm{H}$ and $67.9 \mathrm{MHz}$ for ${ }^{13} \mathrm{C}$ ). Solvents for spectroscopy were distilled and dried with $\mathrm{CaH}_{2}$ under $\mathrm{N}_{2}$ before use.

\section{(2) Preparation of 4, 6, 7, and 8.}

7-Benzhydrylidene-exo,cis-5,6-bis(phenylsulfonyl)norbornene (12). A solution of 11 (5.14 g, $22.3 \mathrm{mmol})$ and (Z)-1,2-bis(phenylsulfonyl)ethene $(7.07 \mathrm{~g}, 22.9 \mathrm{mmol})$ in toluene $(65 \mathrm{~mL})$ was refluxed for $6 \mathrm{~h}$. After removing the toluene in vacuo, the residue was recrystallized from AcOEt $/ n$-hexane to give $5.55 \mathrm{~g}(46 \%)$ of 12 as colorless needles: mp 212-213 ${ }^{\circ} \mathrm{C} ;{ }^{1} \mathrm{H} \mathrm{NMR}\left(\mathrm{CDCl}_{3}\right) \delta_{\mathrm{ppm}} 8.03(4 \mathrm{H}, \mathrm{m}), 7.66(4 \mathrm{H}, \mathrm{m}), 7.56(2 \mathrm{H}, \mathrm{m}), 7.13-7.22(6 \mathrm{H}, \mathrm{m}), 6.83(2 \mathrm{H}, \mathrm{dd}, J$ $=2,2 \mathrm{~Hz}), 6.78$ (4 H, m), 4.29 (2 H, brs), 3.77 (2 H, brs); IR (KBr) 3060, 1490, $1455 \mathrm{~cm}^{-1}$; EIMS m/z $538\left(2, \mathrm{M}^{+}\right), 461$ (63), 230 (100), 77 (54). The stereochemistry of 12 was judged from the negligible coupling constant between the protons at C-1 and C-6 (or C-4 and C-5) using Karplus rule in ${ }^{1} \mathrm{H}$ NMR analyses.

7-Benzhydrylidene-exo,cis-2,3-bis(phenylsulfonyl)norbornane (13). To a solution of 12 (434 $\mathrm{mg}, 805 \mu \mathrm{mol})$ in a mixture of AcOEt $(20 \mathrm{~mL})$ and EtOH $(10 \mathrm{~mL})$ was added $5 \% \mathrm{Pd}-\mathrm{C}(8 \mathrm{mg})$ under $\mathrm{N}_{2}$. The suspended solution was then bubbled with $\mathrm{H}_{2}$ and heated at $40{ }^{\circ} \mathrm{C}$ for $70 \mathrm{~min}$. The reaction mixture was filtered through a pad of celite, and the filtrate concentrated in vacuo. The residue was recrystallized from AcOEt to give $386 \mathrm{mg}(89 \%)$ of $\mathbf{1 3}$ as colorless needles: $\mathrm{mp} 250{ }^{\circ} \mathrm{C}$ (dec.); ${ }^{1} \mathrm{H}$ NMR $\left(\mathrm{CDCl}_{3}\right) \delta_{\mathrm{ppm}} 7.98(4 \mathrm{H}, \mathrm{m}), 7.65(2 \mathrm{H}, \mathrm{m}), 7.54(4 \mathrm{H}, \mathrm{m}), 7.10-7.21(6 \mathrm{H}, \mathrm{m}), 6.82$ (4 H, m), 3.94 (2 H, s), 3.11 (2 H, brs), 3.00 (2 H, m), 1.80 (2 H, m); IR (KBr) 3060, 1600, 1490, $1445 \mathrm{~cm}^{-1}$; EIMS m/z $540\left(1, \mathrm{M}^{+}\right), 399(56), 229(60), 77$ (100).

7-Benzhydrylidenenorbornene (4). ${ }^{15}$ To a solution of $13(380 \mathrm{mg}, 702 \mu \mathrm{mol})$ and $\mathrm{NaH}_{2} \mathrm{PO}_{4} \cdot \mathrm{H}_{2} \mathrm{O}(1.69 \mathrm{~g}, 11.2$ mmol) in $\mathrm{MeOH}(20 \mathrm{~mL})$ was added $3 \% \mathrm{Na}-\mathrm{Hg}(5.6 \mathrm{~g})$ at room temperature under $\mathrm{N}_{2}$. The reaction mixture was vigorously stirred at $40{ }^{\circ} \mathrm{C}$ for $4 \mathrm{~h}$. After filtering the $\mathrm{Hg}$ off, the filtrate was concentrated in vacuo. The residue was chromatographed with silica gel (eluted with 17\% AcOEt in $n$-hexane) to afford $138 \mathrm{mg}(76 \%)$ of 4 as colorless plates: mp 70-71 ${ }^{\circ} \mathrm{C} ;{ }^{1} \mathrm{H} \mathrm{NMR}\left(\mathrm{CDCl}_{3}\right) \delta_{\mathrm{ppm}} 7.17-7.31(6 \mathrm{H}, \mathrm{m}), 7.10(4 \mathrm{H}, \mathrm{m}), 6.28(2 \mathrm{H}, \mathrm{dd}, J=2.2,2.2 \mathrm{~Hz}), 3.35$ (2 H, brs), $1.88(2 \mathrm{H}, \mathrm{m}), 1.18(2 \mathrm{H}, \mathrm{m}) ;{ }^{13} \mathrm{C}$ NMR $\left(\mathrm{CDCl}_{3}\right) \delta_{\mathrm{ppm}} 154.5,141.8,136.0,129.8,127.8,126.3,120.3,43.5,24.6 ; \mathrm{IR}$ (KBr) 3050, 1675, 1600, 1470, $1440 \mathrm{~cm}^{-1}$; EIMS m/z $258\left(85, \mathrm{M}^{+}\right), 178$ (100); Anal. Calcd. for $\mathrm{C}_{20} \mathrm{H}_{18}, \mathrm{C}: 92.98, \mathrm{H}$ : 7.02, Found. C: $92.68, \mathrm{H}: 7.11$.

7-Benzhydrylidenenorbornane (6). To a solution of 4 (372 mg, $1.44 \mathrm{mmol})$ in AcOEt (5 mL) was added 5\% Pd-C (20 mg) under $\mathrm{N}_{2}$. The suspended solution was then bubbled with $\mathrm{H}_{2}$ and stirred at room temperature for $40 \mathrm{~min}$. The reaction mixture was filtered through a pad of celite, and the filtrate concentrated in vacuo. The residue was purified twice by preparative silica gel TLC (eluted with $9 \% \mathrm{CHCl}_{3}$ in $n$-hexane) to give $210 \mathrm{mg}(56 \%)$ of 6 as colorless cubes: mp 84.5-85.5 ${ }^{\circ} \mathrm{C} ;{ }^{1} \mathrm{H}$ NMR $\left(\mathrm{CDCl}_{3}\right) \delta_{\mathrm{ppm}} 7.21-7.29(6 \mathrm{H}, \mathrm{m}), 7.14(4 \mathrm{H}, \mathrm{m}), 2.68(2 \mathrm{H}, \mathrm{tt}, J=2,2 \mathrm{~Hz}), 1.77(4 \mathrm{H}, \mathrm{m})$, $1.43(4 \mathrm{H}, \mathrm{m}) ;{ }^{13} \mathrm{C} \mathrm{NMR}\left(\mathrm{CDCl}_{3}\right) \delta_{\mathrm{ppm}} 150.5,143.0,129.8,127.7,126.6,126.2,37.9,29.0 ; \mathrm{IR}(\mathrm{KBr}) 2940,1595,1490$, $1435 \mathrm{~cm}^{-1}$; EIMS m/z $260\left(100, \mathrm{M}^{+}\right), 232$ (91); HRMS (EI, $70 \mathrm{eV}$ ) Calcd. for $\mathrm{C}_{20} \mathrm{H}_{20}, 260.1565$, Found, 260.1566; Anal. Calcd. for $\mathrm{C}_{20} \mathrm{H}_{20}, \mathrm{C}: 92.26, \mathrm{H}: 7.74$, Found. C: 92.05, H: 7.87.

Dimethyl 7-benzhydrylidenenorbornene-trans-5,6-dicarboxylate (14). A solution of 11 (16.6 g, $71.9 \mathrm{mmol})$ and dimethyl fumarate $(7.11 \mathrm{~g}, 49.3 \mathrm{mmol})$ in benzene $(60 \mathrm{~mL})$ was refluxed for $17 \mathrm{~h}$. After removing the benzene in vacuo, the residue was purified by silica gel chromatography (eluted with 5\% AcOEt in $n$-hexane) and then recrystallized from AcOEt $/ n$-hexane to give $15.0 \mathrm{~g}(81 \%)$ of $\mathbf{1 4}$ as colorless cubes: $\mathrm{mp} 91-92{ }^{\circ} \mathrm{C} ;{ }^{1} \mathrm{H} \mathrm{NMR}\left(\mathrm{CDCl}_{3}\right) \delta_{\mathrm{ppm}} 7.21-7.33(6 \mathrm{H}$, $\mathrm{m}), 7.13(2 \mathrm{H}, \mathrm{m}), 7.02(2 \mathrm{H}, \mathrm{m}), 6.49(1 \mathrm{H}, \mathrm{dd}, J=3.3,5.5 \mathrm{~Hz}), 6.32(1 \mathrm{H}, \mathrm{dd}, J=3.3,5.5 \mathrm{~Hz}), 3.78(1 \mathrm{H}, \mathrm{m}), 3.73(1$ H, dd, $J=4.0,4.0 \mathrm{~Hz}$ ), 3.65 (3 H, s), 3.59 (4 H, brs), 2.88 (1 H, d, J=4.0 Hz); IR (KBr) 3010, 2940, 1730, 1595, 1480, $1430 \mathrm{~cm}^{-1}$; EIMS $\mathrm{m} / \mathrm{z} 374\left(7, \mathrm{M}^{+}\right), 230(100)$.

7-Benzhydrylidene-trans-5,6-bis(hydroxymethyl)norbornene (15). To a solution of 14 (5.18 g, $13.8 \mathrm{mmol})$ in anhydrous ether $(50 \mathrm{~mL})$ was added $\mathrm{LiAlH}_{4}(1.53 \mathrm{~g}, 40.3 \mathrm{mmol})$ at room temperature under $\mathrm{N}_{2}$, and the reaction mixture was stirred for $20 \mathrm{~min}$. The reaction was quenched by adding water $(30 \mathrm{~mL})$ and ether $(100 \mathrm{~mL})$. The resulting reaction mixture was extracted with ether $(200 \mathrm{~mL} \times 2)$ and the combined extract dried over $\mathrm{Na}_{2} \mathrm{SO}_{4}$. After removing the solvent in vacuo, the residue was chromatographed by silica gel (eluted with $40 \% n$-hexane in AcOEt) to give 3.85 $\mathrm{g}(88 \%)$ of 15 as colorless cubes: $\mathrm{mp} 95-96{ }^{\circ} \mathrm{C} ;{ }^{1} \mathrm{H}$ NMR $\left(\mathrm{CDCl}_{3}\right) \delta_{\mathrm{ppm}} 7.22-7.31(6 \mathrm{H}, \mathrm{m}), 7.07-7.13(4 \mathrm{H}, \mathrm{m}), 6.48(1$ $\mathrm{H}, \mathrm{dd}, J=3.3,5.9 \mathrm{~Hz}), 6.22(1 \mathrm{H}, \mathrm{dd}, J=3.3,5.9 \mathrm{~Hz}), 3.68(2 \mathrm{H}, \mathrm{m}), 3.46(1 \mathrm{H}, \mathrm{dt}, J=4.0,9.7 \mathrm{~Hz}), 3.39$ (1 H, brs), 
$3.22(1 \mathrm{H}, \mathrm{dt}, J=4.0,9.7 \mathrm{~Hz}), 3.11(1 \mathrm{H}, \mathrm{brd}, J=2.9 \mathrm{~Hz}), 2.32(1 \mathrm{H}, \mathrm{dd}, J=4,5 \mathrm{~Hz}), 2.27(1 \mathrm{H}, \mathrm{t}, J=5 \mathrm{~Hz}), 2.18-2.24$ $(1 \mathrm{H}, \mathrm{m}), 1.52(1 \mathrm{H}, \mathrm{ddd}, J=5,5,10 \mathrm{~Hz})$; IR $(\mathrm{KBr}) 3350,3050,2900,1600,1490,1440 \mathrm{~cm}^{-1}$; EIMS m/z $318\left(6, \mathrm{M}^{+}\right)$, $230(100)$.

7-Benzhydrylidene-trans-5,6-bis(tosyloxymethyl)norbornene (16). To a solution of 15 (3.04 g, $9.56 \mathrm{mmol})$ in anhydrous pyridine $(6 \mathrm{~mL})$ was added $\mathrm{TsCl}(4.05 \mathrm{~g}, 21.2 \mathrm{mmol})$ at $0{ }^{\circ} \mathrm{C}$ under $\mathrm{N}_{2}$, and the reaction mixture was kept in an ice bath for $5 \mathrm{~h}$. After quenching the reaction by adding $1.2 \mathrm{M} \mathrm{HCl}$ aqueous solution $(100 \mathrm{~mL})$, the reaction mixture was extracted with ether $(100 \mathrm{~mL} \times 6)$. The combined extract was washed with brine and dried over $\mathrm{Na}_{2} \mathrm{SO}_{4}$. After removing the solvent in vacuo, the residue was purified by silica gel chromatography (eluted with 25 to $40 \%$ AcOEt in $n$-hexane) and then recrystallized from AcOEt $/ n$-hexane to give $5.73 \mathrm{~g}(96 \%)$ of 16 as colorless cubes: $\mathrm{mp} 130-131{ }^{\circ} \mathrm{C}$; ${ }^{1} \mathrm{H}$ NMR $\left(\mathrm{CDCl}_{3}\right) \delta_{\mathrm{ppm}} 7.77$ (2 H, AA'XX'), 7.65 (2 H, AA'XX'), 7.24-7.38 (10 H, m), 6.90-6.97 (4 H, m), 6.36 (1 H, $\mathrm{dd}, J=3,6 \mathrm{~Hz}), 6.03(1 \mathrm{H}, \mathrm{dd}, J=3,6 \mathrm{~Hz}), 3.84-3.90(3 \mathrm{H}, \mathrm{m}), 3.63(1 \mathrm{H}, \mathrm{dd}, J=10,10 \mathrm{~Hz}), 3.38$ (1 H, brs), 3.13 (1 $\mathrm{H}, \mathrm{d}, J=2.9 \mathrm{~Hz}), 2.46(3 \mathrm{H}, \mathrm{s}), 2.44(3 \mathrm{H}, \mathrm{s}), 2.13(1 \mathrm{H}, \mathrm{m}), 1.36(1 \mathrm{H}, \mathrm{m})$; IR $(\mathrm{KBr}) 3000,1600,1490,1440 \mathrm{~cm}^{-1}$; FABMS ( $m$-NBA) $m / z 626\left(\mathrm{M}^{+}\right)$.

7-Benzhydrylidene-trans-2,3-bis(tosyloxymethyl)norbornane (17). To a solution of $\mathbf{1 6}(1.01 \mathrm{~g}, 1.62 \mathrm{mmol}) \mathrm{in}$ a mixture of AcOEt $(10 \mathrm{~mL})$ and EtOH $(10 \mathrm{~mL})$ was added $5 \% \mathrm{Pd}-\mathrm{C}(6 \mathrm{mg})$ under $\mathrm{N}_{2}$. The suspended solution was then bubbled with $\mathrm{H}_{2}$ and heated under reflux for $2 \mathrm{~h}$. The reaction mixture was filtered through a pad of celite, and the filtrate concentrated in vacuo. The residue was recrystallized from ether $n$-hexane to give $0.98 \mathrm{~g}(96 \%)$ of $\mathbf{1 7}$ as colorless cubes: $\mathrm{mp} 111-111.5{ }^{\circ} \mathrm{C} ;{ }^{1} \mathrm{H} \mathrm{NMR}_{\left(\mathrm{CDCl}_{3}\right)} \delta_{\mathrm{ppm}} 7.78\left(2 \mathrm{H}, \mathrm{AA}^{\prime} \mathrm{XX}\right), 7.65$ (2 H, AA'XX'), $7.36\left(2 \mathrm{H}, \mathrm{AA}^{\prime} \mathrm{XX}\right)$, $7.31\left(2 \mathrm{H}, \mathrm{AA}^{\prime} \mathrm{XX}\right), 7.22-7.29(6 \mathrm{H}, \mathrm{m}), 6.95-7.01(4 \mathrm{H}, \mathrm{m}), 4.09(1 \mathrm{H}, \mathrm{dd}, J=6.7,9.5 \mathrm{~Hz}), 3.93(1 \mathrm{H}, \mathrm{dd}, J=10.2$, $10.2 \mathrm{~Hz}), 3.77(1 \mathrm{H}, \mathrm{dd}, J=8.3,9.5 \mathrm{~Hz}), 3.64(1 \mathrm{H}, \mathrm{dd}, J=6.7,8.3 \mathrm{~Hz}), 2.37(1 \mathrm{H}, \mathrm{dd}, J=4,4 \mathrm{~Hz}), 2.48(1 \mathrm{H}, \mathrm{d}, J=$ $4.8 \mathrm{~Hz}), 2.46$ (3 H, s), 2.44 (3 H, s), 1.73-1.79 (2 H, m), 1.38-1.56 (3 H, m), 1.23 (1 H, m); IR (KBr) 3050, 2980, 1600 , $1590,1540 \mathrm{~cm}^{-1}$; FABMS ( $m$-NBA) $m / z 628\left(\mathrm{M}^{+}\right)$

7-Benzhydrylidene-2,3-dimethylenenorbornane (7). To a solution of $\mathbf{1 7}$ (763 $\mathrm{mg}, 1.21 \mathrm{mmol})$ in anhydrous DMSO $(10 \mathrm{~mL})$ was added $t$-BuOK $(647 \mathrm{mg}, 5.76 \mathrm{mmol})$ at room temperature under $\mathrm{N}_{2}$. After stirring the reaction mixture for $3 \mathrm{~h}$, the reaction was quenched by adding water. The reaction mixture was extracted with AcOEt and $n$-hexane, and the combined extract dried over $\mathrm{Na}_{2} \mathrm{SO}_{4}$. After removing the solvent in vacuo, the residue was chromatographed with silica gel (eluted with $9 \%$ AcOEt in $n$-hexane) to give $145 \mathrm{mg}(42 \%)$ of 7 as colorless cubes: mp $106-107{ }^{\circ} \mathrm{C}$; ${ }^{1} \mathrm{H} \mathrm{NMR}$ $\left(\mathrm{CDCl}_{3}\right) \delta_{\text {ppm }} 7.23-7.33(6 \mathrm{H}, \mathrm{m}), 7.13(4 \mathrm{H}, \mathrm{m}), 5.17(2 \mathrm{H}, \mathrm{s}), 4.82(2 \mathrm{H}, \mathrm{s}), 3.33(2 \mathrm{H}, \mathrm{dd}, J=2.2,2.2 \mathrm{~Hz}), 1.96(2 \mathrm{H}$, $\mathrm{m}), 1.60(2 \mathrm{H}, \mathrm{m}) ;{ }^{13} \mathrm{C}$ NMR $\left(\mathrm{CD}_{3} \mathrm{OD}\right) \delta_{\mathrm{ppm}} 152.2,147.8,143.3,130.8,129.4,129.0,127.8,100.4,49.5,29.4 ; \mathrm{IR}(\mathrm{KBr})$ 2960, 1595, 1485, $1440 \mathrm{~cm}^{-1}$; EIMS m/z $284\left(42, \mathrm{M}^{+}\right), 256(100)$; HRMS (EI, $\left.70 \mathrm{eV}\right) \mathrm{Calcd}$. for $\mathrm{C}_{22} \mathrm{H}_{20}, 284.1565$, Found, 284.1538; Anal. Calcd. for $\mathrm{C}_{20} \mathrm{H}_{18}, \mathrm{C}: 92.91$, H: 7.09, Found. C: 92.44, H: 7.28.

7-Benzhydrylidene-5,6-dimethylenenorbornene (8). To a solution of $\mathbf{1 6}(3.01 \mathrm{~g}, 4.81 \mathrm{mmol})$ in anhydrous DMSO $(30 \mathrm{~mL})$ was added $t$-BuOK $(1.32 \mathrm{~g}, 11.8 \mathrm{mmol})$ at room temperature under $\mathrm{N}_{2}$. After stirring the reaction mixture for 1 $\mathrm{h}$, the reaction was quenched by adding water. The reaction mixture was extracted with AcOEt and $n$-hexane, and the combined extract dried over $\mathrm{Na}_{2} \mathrm{SO}_{4}$. After removing the solvent in vacuo, the residue was chromatographed with silica gel (eluted with $9 \%$ AcOEt in $n$-hexane) to give $1.24 \mathrm{~g}(91 \%)$ of 8 as colorless plates: mp $93-94{ }^{\circ} \mathrm{C} ;{ }^{1} \mathrm{H} \mathrm{NMR}\left(\mathrm{CDCl}_{3}\right)$ $\delta_{\text {ppm }} 7.26-7.33(6 \mathrm{H}, \mathrm{m}), 7.11(4 \mathrm{H}, \mathrm{m}), 6.47(2 \mathrm{H}, \mathrm{dd}, J=2.2,2.2 \mathrm{~Hz}), 5.25(2 \mathrm{H}, \mathrm{s}), 5.01(2 \mathrm{H}, \mathrm{s}), 3.87(2 \mathrm{H}, \mathrm{dd}, J=$ $2.2,2.2 \mathrm{~Hz}) ;{ }^{13} \mathrm{C} \mathrm{NMR}\left(\mathrm{CDCl}_{3}\right) \delta_{\mathrm{ppm}} 152.7,147.3,140.9,137.1,129.9,127.9,126.7,123.1,101.8,53.4 ; \mathrm{IR}(\mathrm{KBr}) 3050$, 1675, 1640, 1595, 1485, $1440 \mathrm{~cm}^{-1}$; EIMS m/z $282\left(100, \mathrm{M}^{+}\right), 191$ (50); HRMS (EI, $70 \mathrm{eV}$ ) Calcd. for $\mathrm{C}_{22} \mathrm{H}_{18}, 282.1409$, Found, 282.1405; Anal. Calcd. for $\mathrm{C}_{20} \mathrm{H}_{18}$, C: 93.57, H: 6.43, Found. C: 93.04, H: 6.72.

(3) The DFT calculation results of $4,4^{\bullet+}, 4^{2+}, 4^{\bullet-}, 6,6^{\bullet+}, 6^{2+}, 6^{\bullet-}, 7,7^{\bullet+}, 7^{2+}, 7^{\bullet-}, 8,8^{\bullet+}, 8^{2+}, 8^{\bullet-}, 1^{-}, 1^{\bullet}$, and $\mathbf{1}^{+}$.

Table S1. The Cartesian Coordinates (in $\AA$ ) of 4 Optimized by RB3LYP/cc-pVDZ. (The total electronic energy is -772.957060613 Hartree.)

\begin{tabular}{rrrr}
\hline & $\mathrm{X}$ & \multicolumn{1}{c}{$\mathrm{Y}$} & \multicolumn{1}{c}{$\mathrm{Z}$} \\
\hline $\mathrm{C} 1$ & 1.271164 & -0.107533 & -0.053361 \\
$\mathrm{C} 2$ & 2.200986 & -1.312884 & 0.070944 \\
$\mathrm{C} 3$ & 2.379034 & 0.927131 & -0.243669 \\
$\mathrm{C} 4$ & 3.059415 & -1.090237 & -1.170687 \\
$\mathrm{C} 5$ & 3.161159 & 0.236924 & -1.359173 \\
$\mathrm{C} 6$ & 3.069146 & -0.847328 & 1.295634 \\
$\mathrm{C} 7$ & 3.205437 & 0.690707 & 1.070486 \\
$\mathrm{C} 8$ & -0.070948 & 0.003772 & -0.017293 \\
$\mathrm{C} 9$ & -0.954231 & -1.201885 & 0.056716 \\
$\mathrm{C} 10$ & -0.75284 & 1.334614 & -0.068982 \\
$\mathrm{C} 11$ & -0.800358 & -2.279022 & -0.834577 \\
$\mathrm{C} 12$ & -0.382606 & 2.388365 & 0.786051 \\
$\mathrm{C} 13$ & -1.629649 & -3.401749 & -0.755723
\end{tabular}




\begin{tabular}{rrrr} 
C14 & -1.024323 & 3.628601 & 0.720478 \\
C15 & -2.633259 & -3.46833 & 0.214403 \\
C16 & -2.055248 & 3.839533 & -0.198886 \\
C17 & -2.807253 & -2.398182 & 1.098643 \\
C18 & -2.444316 & 2.796666 & -1.046301 \\
C19 & -1.982606 & -1.275019 & 1.015056 \\
C20 & -1.805642 & 1.557481 & -0.976712 \\
H21 & 1.748926 & -2.302545 & 0.188544 \\
H22 & 2.086039 & 1.965682 & -0.427245 \\
H23 & 3.564931 & -1.880042 & -1.729035 \\
H24 & 3.765109 & 0.757493 & -2.104332 \\
H25 & 2.540744 & -1.066968 & 2.23621 \\
H26 & 2.767388 & 1.256795 & 1.907433 \\
H27 & -0.033692 & -2.221974 & -1.610093 \\
H28 & 0.404693 & 2.224126 & 1.524326 \\
H29 & -1.495315 & -4.224372 & -1.462456 \\
H30 & -0.722309 & 4.430429 & 1.398707 \\
H31 & -3.282282 & -4.344907 & 0.276424 \\
H32 & -2.558686 & 4.80771 & -0.249813 \\
H33 & -3.592142 & -2.438021 & 1.857905 \\
H34 & -3.252047 & 2.949211 & -1.766313 \\
H35 & -2.129367 & -0.440976 & 1.704875 \\
H36 & -2.118471 & 0.747993 & -1.639748 \\
H37 & 4.24945 & 1.017506 & 0.9541 \\
H38 & 4.038651 & -1.3669 & 1.322216 \\
\hline & & &
\end{tabular}

Table S2. The Cartesian Coordinates (in $\AA$ ), and Mülliken Spin $(\rho)$ and Charge $(q)$ Densities of $\mathbf{4}^{{ }^{+}}$Obtained by UB3LYP/cc-pVDZ Calculation. (The total electronic energy is -772.698432417 Hartree.)

\begin{tabular}{lrrrrr}
\hline & \multicolumn{1}{c}{$\mathrm{X}$} & \multicolumn{1}{c}{$\mathrm{Y}$} & \multicolumn{1}{c}{$\mathrm{Z}$} & \multicolumn{1}{c}{$\rho$} & \multicolumn{1}{c}{$q$} \\
\hline $\mathrm{C} 1$ & -1.29727 & -0.128938 & -0.0433 & 0.326152 & 0.090285 \\
$\mathrm{C} 2$ & -2.401439 & 0.850164 & 0.32976 & -0.015286 & -0.056689 \\
$\mathrm{C} 3$ & -2.18335 & -1.34256 & -0.279481 & -0.011774 & -0.054047 \\
$\mathrm{C} 4$ & -2.88857 & -0.010951 & 1.492895 & 0.081438 & 0.079743 \\
$\mathrm{C} 5$ & -2.766522 & -1.31261 & 1.129575 & 0.081444 & 0.065475 \\
$\mathrm{C} 6$ & -3.409388 & 0.702557 & -0.851183 & -0.00029 & 0.070213 \\
$\mathrm{C} 7$ & -3.247192 & -0.78443 & -1.276765 & -0.000523 & 0.080562 \\
$\mathrm{C} 8$ & 0.098115 & 0.009852 & -0.018591 & 0.145252 & 0.055592 \\
$\mathrm{C} 9$ & 0.712951 & 1.339958 & 0.042511 & 0.033372 & -0.029411 \\
$\mathrm{C} 10$ & 0.980681 & -1.161162 & -0.078388 & 0.026036 & -0.038849 \\
$\mathrm{C} 11$ & 1.897525 & 1.531933 & 0.802923 & 0.072074 & 0.038088 \\
$\mathrm{C} 12$ & 2.15855 & -1.113369 & -0.870194 & 0.074316 & 0.037866 \\
$\mathrm{C} 13$ & 2.489709 & 2.787248 & 0.888799 & -0.037605 & 0.057161 \\
$\mathrm{C} 14$ & 3.003517 & -2.21565 & -0.952167 & -0.038441 & 0.05686 \\
$\mathrm{C} 15$ & 1.938514 & 3.872093 & 0.194532 & 0.116877 & 0.063171 \\
$\mathrm{C} 16$ & 2.713995 & -3.375928 & -0.223785 & 0.107712 & 0.061742 \\
$\mathrm{C} 17$ & 0.782902 & 3.697236 & -0.583392 & -0.025903 & 0.057388 \\
$\mathrm{C} 18$ & 1.566863 & -3.432699 & 0.584033 & -0.017096 & 0.055485 \\
$\mathrm{C} 19$ & 0.169999 & 2.452541 & -0.651667 & 0.055094 & 0.029711 \\
$\mathrm{C} 20$ & 0.703319 & -2.345935 & 0.647717 & 0.044553 & 0.03415 \\
$\mathrm{H} 21$ & 2.317612 & 0.693614 & 1.359996 & -0.002962 & 0.000931 \\
$\mathrm{H} 22$ & 2.375973 & -0.217629 & -1.453679 & -0.003067 & -0.001096 \\
$\mathrm{H} 23$ & 3.382063 & 2.925771 & 1.50159 & 0.001905 & 0.012678 \\
$\mathrm{H} 24$ & 3.889727 & -2.174411 & -1.587739 & 0.00192 & 0.012 \\
& & & & &
\end{tabular}




\begin{tabular}{rrrrrr} 
H25 & 2.409195 & 4.855381 & 0.255863 & -0.004724 & 0.018322 \\
H26 & 3.382048 & -4.237651 & -0.281054 & -0.004378 & 0.017493 \\
H27 & 0.369438 & 4.538167 & -1.142819 & 0.000856 & 0.010866 \\
H28 & 1.357114 & -4.328936 & 1.170566 & 0.00063 & 0.009941 \\
H29 & -0.704201 & 2.318624 & -1.289731 & -0.002494 & -0.012292 \\
H30 & -0.163572 & -2.385047 & 1.308281 & -0.003188 & -0.019023 \\
H31 & -2.119725 & 1.871227 & 0.600259 & 0.00137 & 0.003724 \\
H32 & -1.706063 & -2.277075 & -0.585224 & 0.000844 & 0.002885 \\
H33 & -3.266781 & 0.382635 & 2.437436 & -0.002422 & 0.02131 \\
H34 & -3.03357 & -2.191484 & 1.718666 & -0.002304 & 0.020938 \\
H35 & -4.429026 & 0.953284 & -0.527971 & 0.000647 & 0.038658 \\
H36 & -3.142642 & 1.38777 & -1.670124 & -0.000152 & 0.035087 \\
H37 & -4.180803 & -1.359864 & -1.209178 & 0.00038 & 0.038677 \\
H38 & -2.874241 & -0.870471 & -2.308126 & -0.000263 & 0.034406 \\
\hline
\end{tabular}

Table S3. The Cartesian Coordinates (in $\AA$ ), and Mülliken Charge $(q)$ Densities of $4^{2+}$ Obtained by RB3LYP/cc-pVDZ Calculation. (The total electronic energy is -772.308166902 Hartree.)

\begin{tabular}{rrrrr}
\hline & \multicolumn{1}{c}{$\mathrm{X}$} & \multicolumn{1}{c}{$\mathrm{Y}$} & \multicolumn{1}{c}{$\mathrm{Z}$} & \multicolumn{1}{c}{$q$} \\
\hline $\mathrm{C} 1$ & 0.851487 & -1.057776 & -0.084796 & -0.113088 \\
$\mathrm{C} 2$ & 2.025481 & -1.389337 & -1.016314 & -0.003645 \\
$\mathrm{C} 3$ & 1.165263 & -2.020451 & 1.099146 & 0.010709 \\
$\mathrm{C} 4$ & 0.974449 & -2.428977 & -1.211471 & 0.134935 \\
$\mathrm{C} 5$ & 0.468182 & -2.797204 & 0.045577 & 0.141529 \\
$\mathrm{C} 6$ & 3.249787 & -1.791423 & -0.178871 & 0.069925 \\
$\mathrm{C} 7$ & 2.689325 & -2.160871 & 1.212815 & 0.066254 \\
$\mathrm{C} 8$ & -0.137352 & 0.060446 & -0.098585 & 0.141779 \\
$\mathrm{C} 9$ & 0.41818 & 1.377254 & -0.098155 & 0.001496 \\
$\mathrm{C} 10$ & -1.55828 & -0.166452 & -0.009649 & -0.025305 \\
$\mathrm{C} 11$ & -0.365711 & 2.483905 & -0.571641 & 0.032712 \\
$\mathrm{C} 12$ & -2.341059 & 0.694091 & 0.825093 & 0.027554 \\
$\mathrm{C} 13$ & 0.157104 & 3.767009 & -0.577548 & 0.07614 \\
$\mathrm{C} 14$ & -3.708962 & 0.497511 & 0.961196 & 0.074465 \\
$\mathrm{C} 15$ & 1.460795 & 3.994235 & -0.108438 & 0.09829 \\
$\mathrm{C} 16$ & -4.343798 & -0.527574 & 0.246988 & 0.08835 \\
$\mathrm{C} 17$ & 2.257153 & 2.925411 & 0.353247 & 0.075668 \\
$\mathrm{C} 18$ & -3.599472 & -1.372669 & -0.598653 & 0.070679 \\
$\mathrm{C} 19$ & 1.758026 & 1.637131 & 0.340927 & 0.046448 \\
$\mathrm{C} 20$ & -2.227524 & -1.215902 & -0.708867 & 0.004868 \\
$\mathrm{H} 21$ & -1.351747 & 2.300696 & -0.998168 & 0.038228 \\
$\mathrm{H} 22$ & -1.843869 & 1.457643 & 1.424125 & 0.029135 \\
$\mathrm{H} 23$ & -0.436111 & 4.596055 & -0.966739 & 0.05097 \\
$\mathrm{H} 24$ & -4.284822 & 1.136036 & 1.633271 & 0.048264 \\
$\mathrm{H} 25$ & 1.870193 & 5.007251 & -0.114493 & 0.062334 \\
$\mathrm{H} 26$ & -5.421704 & -0.674077 & 0.345839 & 0.058156 \\
$\mathrm{H} 27$ & 3.264733 & 3.120183 & 0.723839 & 0.044413 \\
$\mathrm{H} 28$ & -4.107971 & -2.144261 & -1.179274 & 0.043317 \\
$\mathrm{H} 29$ & 2.374689 & 0.82756 & 0.731325 & -0.001549 \\
$\mathrm{H} 30$ & -1.686633 & -1.842489 & -1.416644 & -0.012639 \\
$\mathrm{H} 31$ & 2.176997 & -0.749883 & -1.890007 & 0.05405 \\
$\mathrm{H} 32$ & 0.584463 & -1.904033 & 2.018339 & 0.05676 \\
$\mathrm{H} 33$ & 0.464057 & -2.629124 & -2.158229 & 0.102033 \\
$\mathrm{H} 34$ & -0.488525 & -3.305484 & 0.188006 & 0.092839 \\
$\mathrm{H} 35$ & 3.776857 & -2.624195 & -0.665286 & 0.081562
\end{tabular}




\begin{tabular}{rrrrr} 
H36 & 3.954686 & -0.94939 & -0.131451 & 0.071713 \\
H37 & 2.941307 & -3.18538 & 1.522456 & 0.086094 \\
H38 & 3.054956 & -1.487246 & 2.001087 & 0.074556 \\
\hline
\end{tabular}

Table S4. The Cartesian Coordinates (in $\AA$ ), and Mülliken Spin $(\rho)$ and Charge $(q)$ Densities of $4^{*-}$ Obtained by UB3LYP/cc-pVDZ Calculation. (The total electronic energy is -772.9432426737 Hartree.)

\begin{tabular}{|c|c|c|c|c|c|}
\hline & X & $\mathrm{Y}$ & $\mathrm{Z}$ & $\rho$ & $q$ \\
\hline $\mathrm{C} 1$ & -0.342849 & 1.195047 & -0.056731 & 0.492219 & -0.012174 \\
\hline $\mathrm{C} 2$ & -1.682028 & 1.848154 & -0.388257 & -0.028178 & -0.052063 \\
\hline C3 & 0.288166 & 2.471294 & 0.503848 & -0.024896 & -0.03586 \\
\hline $\mathrm{C} 4$ & -1.994695 & 2.5755 & 0.924381 & 0.035743 & 0.039628 \\
\hline $\mathrm{C} 5$ & -0.814994 & 2.938107 & 1.460418 & 0.004035 & 0.042229 \\
\hline C6 & -1.194794 & 2.980097 & -1.370743 & 0.007277 & 0.092453 \\
\hline $\mathrm{C} 7$ & 0.159322 & 3.429733 & -0.739806 & 0.009808 & 0.083408 \\
\hline $\mathrm{C} 8$ & 0.060688 & -0.153236 & -0.024346 & 0.001138 & -0.018779 \\
\hline C9 & -0.938762 & -1.228771 & -0.042233 & 0.057097 & -0.038029 \\
\hline $\mathrm{C} 10$ & 1.485289 & -0.496272 & 0.010421 & 0.059204 & -0.017243 \\
\hline $\mathrm{C} 11$ & -2.201398 & -1.099895 & 0.610827 & 0.058072 & 0.003165 \\
\hline $\mathrm{C} 12$ & 2.486627 & 0.365871 & -0.532577 & 0.068315 & -0.003014 \\
\hline $\mathrm{C} 13$ & -3.172715 & -2.095853 & 0.548598 & -0.026103 & 0.032885 \\
\hline C14 & 3.845894 & 0.078071 & -0.437905 & -0.030995 & 0.036392 \\
\hline C15 & -2.949997 & -3.285099 & -0.165272 & 0.128084 & -0.012341 \\
\hline C16 & 4.30017 & -1.086035 & 0.201176 & 0.122507 & -0.011949 \\
\hline C17 & -1.712006 & -3.440704 & -0.816131 & -0.020721 & 0.032796 \\
\hline C18 & 3.33502 & -1.958407 & 0.740378 & -0.011593 & 0.031488 \\
\hline C19 & -0.741571 & -2.446099 & -0.762172 & 0.053312 & -0.003782 \\
\hline $\mathrm{C} 20$ & 1.977381 & -1.673048 & 0.652812 & 0.039102 & -0.006602 \\
\hline $\mathrm{H} 21$ & -2.480601 & 1.219336 & -0.798366 & 0.000998 & -0.062798 \\
\hline $\mathrm{H} 22$ & 1.2976 & 2.409956 & 0.927333 & 0.000521 & -0.064184 \\
\hline $\mathrm{H} 23$ & -2.99205 & 2.84827 & 1.281923 & 0.000963 & -0.07506 \\
\hline $\mathrm{H} 24$ & -0.659683 & 3.5558 & 2.350598 & 0.004311 & -0.077335 \\
\hline $\mathrm{H} 25$ & -1.045125 & 2.556431 & -2.376696 & 0.000135 & -0.019306 \\
\hline $\mathrm{H} 26$ & 0.999046 & 3.264967 & -1.434245 & -0.000353 & -0.014861 \\
\hline $\mathrm{H} 27$ & -2.388169 & -0.204422 & 1.206841 & -0.003444 & -0.068057 \\
\hline $\mathrm{H} 28$ & 2.163543 & 1.25671 & -1.074011 & -0.000802 & -0.079017 \\
\hline H29 & -4.119185 & -1.951637 & 1.082014 & 0.001306 & -0.080313 \\
\hline H30 & 4.568974 & 0.76934 & -0.886007 & 0.001772 & -0.082408 \\
\hline H31 & -3.714623 & -4.065051 & -0.214607 & -0.006538 & -0.089441 \\
\hline H32 & 5.36794 & -1.307516 & 0.279841 & -0.006126 & -0.09074 \\
\hline H33 & -1.509905 & -4.352824 & -1.388844 & 0.000415 & -0.078758 \\
\hline H34 & 3.65477 & -2.873242 & 1.251825 & 0.000007 & -0.079397 \\
\hline H35 & 0.198448 & -2.587271 & -1.300685 & -0.00289 & -0.064233 \\
\hline H36 & 1.259287 & -2.358784 & 1.10774 & -0.002257 & -0.061015 \\
\hline H37 & 0.162023 & 4.497987 & -0.457241 & 0.007832 & -0.048484 \\
\hline H38 & -1.918777 & 3.811219 & -1.457532 & 0.010724 & -0.0472 \\
\hline
\end{tabular}

Table S5. The Cartesian Coordinates (in $\AA$ ) of 6 Optimized by RB3LYP/cc-pVDZ. (The total electronic energy is -774.195172177 Hartree.)

\begin{tabular}{rrrr}
\hline & \multicolumn{1}{c}{$\mathrm{X}$} & \multicolumn{1}{c}{$\mathrm{Y}$} & \multicolumn{1}{c}{$\mathrm{Z}$} \\
\hline $\mathrm{C} 1$ & 1.24092 & -0.000072 & -0.000009 \\
$\mathrm{C} 2$ & 2.253868 & 1.127576 & -0.157402 \\
$\mathrm{C} 3$ & 2.253758 & -1.127816 & 0.157402 \\
$\mathrm{C} 4$ & 3.081404 & 0.595934 & -1.358322
\end{tabular}




\begin{tabular}{rrrr} 
C5 & 3.104002 & -0.951592 & -1.126915 \\
C6 & 3.104079 & 0.95127 & 1.126926 \\
C7 & 3.081331 & -0.596253 & 1.358332 \\
C8 & -0.108108 & -0.000002 & -0.000012 \\
C9 & -0.897819 & 1.270223 & -0.063175 \\
C10 & -0.897962 & -1.270137 & 0.063163 \\
C11 & -1.9366 & 1.415143 & -1.002206 \\
C12 & -1.936775 & -1.41492 & 1.00218 \\
C13 & -2.674413 & 2.597474 & -1.081108 \\
C14 & -2.674725 & -2.597164 & 1.081095 \\
C15 & -2.402489 & 3.658117 & -0.210407 \\
C16 & -2.40291 & -3.657856 & 0.210419 \\
C17 & -1.388112 & 3.52235 & 0.741037 \\
C18 & -1.388506 & -3.522222 & -0.741016 \\
C19 & -0.645762 & 2.340114 & 0.814352 \\
C20 & -0.64602 & -2.340073 & -0.814343 \\
H21 & -2.159908 & 0.59003 & -1.682137 \\
H22 & -2.159998 & -0.589769 & 1.682092 \\
H23 & -3.468421 & 2.691129 & -1.82604 \\
H24 & -3.468755 & -2.690714 & 1.826017 \\
H25 & -2.984033 & 4.581105 & -0.267998 \\
H26 & -2.984561 & -4.580776 & 0.268019 \\
H27 & -1.177133 & 4.337631 & 1.437428 \\
H28 & -1.177613 & -4.337539 & -1.437391 \\
H29 & 0.129183 & 2.233028 & 1.575911 \\
H30 & 0.128944 & -2.233089 & -1.575897 \\
H31 & 1.85603 & 2.137306 & -0.306234 \\
H32 & 1.855823 & -2.137508 & 0.306231 \\
H33 & 2.584414 & 0.846797 & -2.307961 \\
H34 & 2.650907 & -1.486754 & -1.976064 \\
H35 & 4.123315 & 1.348436 & 0.996482 \\
H36 & 2.651023 & 1.486474 & 1.976069 \\
H37 & 4.089101 & -1.040622 & 1.384974 \\
H38 & 2.584305 & -0.847069 & 2.307964 \\
H39 & 4.089217 & 1.040207 & -1.384952 \\
H40 & 4.123198 & -1.348856 & -0.996458 \\
\hline & & & \\
\hline
\end{tabular}

Table S6. The Cartesian Coordinates (in $\AA$ ), and Mülliken Spin $(\rho)$ and Charge $(q)$ Densities of $\mathbf{6}^{\circ+}$ Obtained by UB3LYP/cc-pVDZ Calculation. (The total electronic energy is -773.932880153 Hartree.)

\begin{tabular}{rrrrrr}
\hline & \multicolumn{1}{c}{$\mathrm{X}$} & \multicolumn{1}{c}{$\mathrm{Y}$} & \multicolumn{1}{c}{$\mathrm{Z}$} & \multicolumn{1}{c}{$\rho$} & \multicolumn{1}{c}{$q$} \\
\hline $\mathrm{C} 1$ & 1.265798 & -0.000302 & -0.000015 & 0.408731 & 0.108906 \\
$\mathrm{C} 2$ & 2.254158 & -1.112348 & 0.27002 & -0.019294 & -0.090831 \\
$\mathrm{C} 3$ & 2.254606 & 1.111351 & -0.270019 & -0.019294 & -0.090846 \\
$\mathrm{C} 4$ & 3.068647 & -0.451239 & 1.422804 & 0.017256 & 0.07008 \\
$\mathrm{C} 5$ & 3.116983 & 1.06011 & 1.021762 & 0.013872 & 0.056559 \\
$\mathrm{C} 6$ & 3.116592 & -1.06145 & -1.021738 & 0.013873 & 0.056559 \\
$\mathrm{C} 7$ & 3.068859 & 0.449917 & -1.422785 & 0.017257 & 0.070083 \\
$\mathrm{C} 8$ & -0.138859 & 0 & -0.000018 & 0.090298 & 0.033522 \\
$\mathrm{C} 9$ & -0.897581 & -1.253002 & 0.060456 & 0.053063 & -0.030901 \\
$\mathrm{C} 10$ & -0.896992 & 1.253356 & -0.060472 & 0.053067 & -0.030903 \\
$\mathrm{C} 11$ & -2.086367 & -1.3153 & 0.839473 & 0.075951 & 0.040723 \\
$\mathrm{C} 12$ & -2.085775 & 1.316224 & -0.839447 & 0.075953 & 0.040726 \\
$\mathrm{C} 13$ & -2.81796 & -2.494317 & 0.925229 & -0.040283 & 0.057983
\end{tabular}




\begin{tabular}{rrrrrr} 
C14 & -2.8168 & 2.495595 & -0.925179 & -0.040284 & 0.057984 \\
C15 & -2.405291 & -3.627597 & 0.212342 & 0.126183 & 0.065621 \\
C16 & -2.40355 & 3.628681 & -0.21232 & 0.126191 & 0.065623 \\
C17 & -1.247526 & -3.579435 & -0.582932 & -0.019133 & 0.056687 \\
C18 & -1.245763 & 3.57997 & 0.582891 & -0.019137 & 0.056686 \\
C19 & -0.495086 & -2.414807 & -0.649314 & 0.047114 & 0.040678 \\
C20 & -0.493885 & 2.414977 & 0.649248 & 0.04712 & 0.040678 \\
H21 & -2.396294 & -0.440852 & 1.413144 & -0.003133 & 0.001889 \\
H22 & -2.39615 & 0.441922 & -1.413099 & -0.003133 & 0.00189 \\
H23 & -3.709921 & -2.536556 & 1.552536 & 0.001886 & 0.014426 \\
H24 & -3.708768 & 2.538262 & -1.552447 & 0.001886 & 0.014427 \\
H25 & -2.983512 & -4.551739 & 0.274561 & -0.00504 & 0.021083 \\
H26 & -2.981324 & 4.553104 & -0.274524 & -0.005041 & 0.021084 \\
H27 & -0.942042 & -4.457618 & -1.154321 & 0.000571 & 0.013132 \\
H28 & -0.939817 & 4.458016 & 1.154246 & 0.000571 & 0.013132 \\
H29 & 0.382092 & -2.377636 & -1.295208 & -0.002534 & -0.013661 \\
H30 & 0.383324 & 2.377391 & 1.295081 & -0.002535 & -0.013661 \\
H31 & 1.850571 & -2.095948 & 0.527033 & 0.000339 & -0.004672 \\
H32 & 1.85141 & 2.095112 & -0.527039 & 0.000339 & -0.004667 \\
H33 & 2.559134 & -0.592358 & 2.387271 & -0.000464 & 0.02632 \\
H34 & 2.695372 & 1.69733 & 1.813728 & -0.000247 & 0.030603 \\
H35 & 4.135055 & -1.423908 & -0.821861 & 0.001485 & 0.035768 \\
H36 & 2.694754 & -1.698507 & -1.813714 & -0.000247 & 0.030601 \\
H37 & 4.063123 & 0.912259 & -1.503248 & 0.002885 & 0.0373 \\
H38 & 2.559423 & 0.591236 & -2.387263 & -0.000464 & 0.02632 \\
H39 & 4.062726 & -0.913974 & 1.50329 & 0.002884 & 0.0373 \\
H40 & 4.135594 & 1.422169 & 0.821916 & 0.001485 & 0.035768 \\
\hline
\end{tabular}

Table S7. The Cartesian Coordinates (in $\AA$ ), and Mülliken Charge $(q)$ Densities of $\mathbf{6}^{2+}$ Obtained by RB3LYP/cc-pVDZ Calculation. (The total electronic energy is -773.515404437 Hartree.)

\begin{tabular}{rrrrr}
\hline & \multicolumn{1}{c}{$\mathrm{X}$} & \multicolumn{1}{c}{$\mathrm{Y}$} & \multicolumn{1}{c}{$\mathrm{Z}$} & \multicolumn{1}{c}{$\mathrm{q}$} \\
\hline $\mathrm{C} 1$ & 1.310405 & -0.000407 & -0.00006 & 0.155236 \\
$\mathrm{C} 2$ & 2.256071 & -1.097011 & 0.347622 & -0.108132 \\
$\mathrm{C} 3$ & 2.256644 & 1.095742 & -0.347612 & -0.108175 \\
$\mathrm{C} 4$ & 3.049412 & -0.332742 & 1.475751 & 0.064186 \\
$\mathrm{C} 5$ & 3.156852 & 1.129482 & 0.927496 & 0.053839 \\
$\mathrm{C} 6$ & 3.156441 & -1.131181 & -0.927361 & 0.053845 \\
$\mathrm{C} 7$ & 3.049763 & 0.331091 & -1.475638 & 0.064199 \\
$\mathrm{C} 8$ & -0.146336 & -0.000005 & -0.000059 & 0.085454 \\
$\mathrm{C} 9$ & -0.88032 & -1.239593 & 0.073202 & -0.024594 \\
$\mathrm{C} 10$ & -0.879585 & 1.240018 & -0.073268 & -0.024563 \\
$\mathrm{C} 11$ & -2.132765 & -1.26538 & 0.790074 & 0.047924 \\
$\mathrm{C} 12$ & -2.132175 & 1.266516 & -0.78986 & 0.04793 \\
$\mathrm{C} 13$ & -2.886268 & -2.423864 & 0.85656 & 0.079828 \\
$\mathrm{C} 14$ & -2.884964 & 2.425468 & -0.856294 & 0.079831 \\
$\mathrm{C} 15$ & -2.436276 & -3.583173 & 0.200099 & 0.10291 \\
$\mathrm{C} 16$ & -2.43408 & 3.584566 & -0.200073 & 0.10292 \\
$\mathrm{C} 17$ & -1.216763 & -3.582591 & -0.522111 & 0.077314 \\
$\mathrm{C} 18$ & -1.214379 & 3.583305 & 0.521818 & 0.077314 \\
$\mathrm{C} 19$ & -0.438482 & -2.443977 & -0.56844 & 0.052541 \\
$\mathrm{C} 20$ & -0.436805 & 2.444207 & 0.568086 & 0.052536 \\
$\mathrm{H} 21$ & -2.43459 & -0.385993 & 1.36119 & 0.03905
\end{tabular}




\begin{tabular}{rrrrr} 
H22 & -2.434691 & 0.387255 & -1.360805 & 0.039056 \\
H23 & -3.808421 & -2.448152 & 1.43955 & 0.05677 \\
H24 & -3.807242 & 2.450272 & -1.439064 & 0.056772 \\
H25 & -3.022806 & -4.503352 & 0.26198 & 0.071955 \\
H26 & -3.020042 & 4.50511 & -0.261922 & 0.071957 \\
H27 & -0.899456 & -4.486028 & -1.045809 & 0.056293 \\
H28 & -0.896359 & 4.486611 & 1.045312 & 0.056289 \\
H29 & 0.47469 & -2.444441 & -1.162788 & 0.013663 \\
H30 & 0.476547 & 2.444175 & 1.162162 & 0.013658 \\
H31 & 1.840203 & -2.051451 & 0.680016 & 0.027692 \\
H32 & 1.841268 & 2.050382 & -0.680052 & 0.027701 \\
H33 & 2.506936 & -0.370567 & 2.431136 & 0.054097 \\
H34 & 2.791794 & 1.863078 & 1.660547 & 0.061836 \\
H35 & 4.180083 & -1.424015 & -0.657699 & 0.072245 \\
H36 & 2.791146 & -1.864609 & -1.660461 & 0.06183 \\
H37 & 4.017552 & 0.83116 & -1.621693 & 0.080221 \\
H38 & 2.507424 & 0.369169 & -2.43109 & 0.054097 \\
H39 & 4.016945 & -0.83327 & 1.621924 & 0.080224 \\
H40 & 4.180669 & 1.421839 & 0.65798 & 0.072249 \\
\hline
\end{tabular}

Table S8. The Cartesian Coordinates (in $\AA$ ), and Mülliken Spin $(\rho)$ and Charge $(q)$ Densities of $6^{-}$Obtained by UB3LYP/cc-pVDZ Calculation. (The total electronic energy is -774.182295427 Hartree.)

\begin{tabular}{rrrrrr}
\hline & \multicolumn{1}{c}{$\mathrm{X}$} & \multicolumn{1}{c}{$\mathrm{Y}$} & \multicolumn{1}{c}{$\mathrm{Z}$} & \multicolumn{1}{c}{$\rho$} & \multicolumn{1}{c}{$q$} \\
\hline $\mathrm{C} 1$ & -1.214994 & -0.000499 & -0.000063 & 0.469686 & -0.037005 \\
$\mathrm{C} 2$ & -2.236518 & -1.053008 & -0.408344 & -0.02752 & -0.06374 \\
$\mathrm{C} 3$ & -2.237286 & 1.051196 & 0.408356 & -0.02752 & -0.063732 \\
$\mathrm{C} 4$ & -3.076517 & -0.270288 & -1.460115 & 0.013192 & 0.086932 \\
$\mathrm{C} 5$ & -3.119201 & 1.177422 & -0.869153 & 0.011653 & 0.07634 \\
$\mathrm{C} 6$ & -3.118126 & -1.179933 & 0.869299 & 0.011652 & 0.076344 \\
$\mathrm{C} 7$ & -3.076509 & 0.267811 & 1.460254 & 0.013194 & 0.086931 \\
$\mathrm{C} 8$ & 0.194301 & 0.000057 & -0.000137 & 0.012066 & -0.029427 \\
$\mathrm{C} 9$ & 0.943313 & -1.262557 & -0.026965 & 0.058738 & -0.025306 \\
$\mathrm{C} 10$ & 0.942257 & 1.263289 & 0.026833 & 0.058722 & -0.025308 \\
$\mathrm{C} 11$ & 2.1853 & -1.398643 & -0.71873 & 0.045823 & -0.00271 \\
$\mathrm{C} 12$ & 2.183974 & 1.400455 & 0.71887 & 0.045835 & -0.002712 \\
$\mathrm{C} 13$ & 2.864466 & -2.609173 & -0.795164 & -0.015086 & 0.031655 \\
$\mathrm{C} 14$ & 2.862088 & 2.611568 & 0.795418 & -0.015097 & 0.03166 \\
$\mathrm{C} 15$ & 2.348336 & -3.773077 & -0.19353 & 0.128849 & -0.011935 \\
$\mathrm{C} 16$ & 2.34512 & 3.775005 & 0.193609 & 0.12883 & -0.011934 \\
$\mathrm{C} 17$ & 1.129618 & -3.669409 & 0.495404 & -0.030362 & 0.033924 \\
$\mathrm{C} 18$ & 1.126662 & 3.67027 & -0.495629 & -0.030345 & 0.033922 \\
$\mathrm{C} 19$ & 0.448326 & -2.457492 & 0.577845 & 0.066631 & 0.003082 \\
$\mathrm{C} 20$ & 0.446417 & 2.457772 & -0.578172 & 0.066594 & 0.003104 \\
$\mathrm{H} 21$ & 2.601237 & -0.521708 & -1.219846 & -0.002543 & -0.062084 \\
$\mathrm{H} 22$ & 2.600535 & 0.523898 & 1.220132 & -0.002544 & -0.06208 \\
$\mathrm{H} 23$ & 3.81009 & -2.655931 & -1.346776 & 0.000142 & -0.078839 \\
$\mathrm{H} 24$ & 3.80754 & 2.659156 & 1.347255 & 0.000143 & -0.078835 \\
$\mathrm{H} 25$ & 2.878747 & -4.72661 & -0.262517 & -0.006488 & -0.089721 \\
$\mathrm{H} 26$ & 2.87471 & 4.728988 & 0.262681 & -0.006487 & -0.089716 \\
$\mathrm{H} 27$ & 0.709497 & -4.551285 & 0.992435 & 0.001702 & -0.081673 \\
$\mathrm{H} 28$ & 0.705918 & 4.551763 & -0.992813 & 0.001701 & -0.081668 \\
$\mathrm{H} 29$ & -0.474979 & -2.402822 & 1.156606 & -0.002317 & -0.079059 \\
$\mathrm{H} 30$ & -0.476698 & 2.402287 & -1.157161 & -0.002316 & -0.07906
\end{tabular}




\begin{tabular}{rrrrrr} 
H31 & -1.851518 & -2.009709 & -0.782839 & 0.000957 & -0.064973 \\
H32 & -1.852994 & 2.00821 & 0.782784 & 0.000956 & -0.064971 \\
H33 & -2.564338 & -0.284614 & -2.435386 & -0.000288 & -0.023435 \\
H34 & -2.689243 & 1.909037 & -1.572276 & -0.000133 & -0.019543 \\
H35 & -4.148472 & -1.512398 & 0.64338 & 0.005596 & -0.047136 \\
H36 & -2.687454 & -1.911191 & 1.572357 & -0.000132 & -0.019539 \\
H37 & -4.087527 & 0.693204 & 1.60816 & 0.010606 & -0.048595 \\
H38 & -2.56422 & 0.282548 & 2.43546 & -0.000289 & -0.023432 \\
H39 & -4.087218 & -0.696478 & -1.607892 & 0.010606 & -0.04859 \\
H40 & -4.149776 & 1.509054 & -0.643056 & 0.005594 & -0.04714 \\
\hline
\end{tabular}

Table S9. The Cartesian Coordinates (in $\AA$ ) of 7 Optimized by RB3LYP/cc-pVDZ. (The total electronic energy is -850.370953169 Hartree.)

\begin{tabular}{rrrr}
\hline & \multicolumn{1}{c}{$\mathrm{X}$} & \multicolumn{1}{c}{$\mathrm{Y}$} & \multicolumn{1}{c}{$\mathrm{Z}$} \\
\hline $\mathrm{C} 1$ & -0.890096 & -0.112309 & 0.330367 \\
$\mathrm{C} 2$ & -1.815142 & -1.325594 & 0.299379 \\
$\mathrm{C} 3$ & -1.971413 & 0.9237 & 0.623827 \\
$\mathrm{C} 4$ & -2.832234 & -0.844508 & -0.736178 \\
$\mathrm{C} 5$ & -2.939021 & 0.629311 & -0.518692 \\
$\mathrm{C} 6$ & -2.49802 & -1.217693 & 1.696372 \\
$\mathrm{C} 7$ & -2.591175 & 0.325331 & 1.926102 \\
$\mathrm{C} 8$ & 0.438931 & 0.010079 & 0.136864 \\
$\mathrm{C} 9$ & 1.320955 & -1.184302 & -0.045765 \\
$\mathrm{C} 10$ & 1.103943 & 1.349455 & 0.0838 \\
$\mathrm{C} 11$ & 2.237389 & -1.232069 & -1.113484 \\
$\mathrm{C} 12$ & 2.255283 & 1.603657 & 0.852533 \\
$\mathrm{C} 13$ & 3.056026 & -2.345693 & -1.307569 \\
$\mathrm{C} 14$ & 2.879851 & 2.851584 & 0.818083 \\
$\mathrm{C} 15$ & 2.990444 & -3.431488 & -0.42794 \\
$\mathrm{C} 16$ & 2.377211 & 3.868873 & -0.000157 \\
$\mathrm{C} 17$ & 2.100416 & -3.390656 & 0.648513 \\
$\mathrm{C} 18$ & 1.246647 & 3.624814 & -0.78451 \\
$\mathrm{C} 19$ & 1.276451 & -2.277512 & 0.838197 \\
$\mathrm{C} 20$ & 0.617009 & 2.377319 & -0.744156 \\
$\mathrm{H} 21$ & 2.298371 & -0.387193 & -1.802742 \\
$\mathrm{H} 22$ & 2.658319 & 0.812361 & 1.488442 \\
$\mathrm{H} 23$ & 3.750962 & -2.365686 & -2.150565 \\
$\mathrm{H} 24$ & 3.765956 & 3.030484 & 1.431943 \\
$\mathrm{H} 25$ & 3.635627 & -4.300394 & -0.576561 \\
$\mathrm{H} 26$ & 2.86971 & 4.843482 & -0.032083 \\
$\mathrm{H} 27$ & 2.052062 & -4.22566 & 1.351695 \\
$\mathrm{H} 28$ & 0.855091 & 4.406907 & -1.439584 \\
$\mathrm{H} 29$ & 0.60234 & -2.24208 & 1.696176 \\
$\mathrm{H} 30$ & -0.252752 & 2.186331 & -1.376006 \\
$\mathrm{H} 31$ & -1.372725 & -2.300892 & 0.074328 \\
$\mathrm{H} 32$ & -1.668221 & 1.971391 & 0.710833 \\
$\mathrm{C} 33$ & -3.462768 & -1.603939 & -1.641428 \\
$\mathrm{C} 34$ & -3.691633 & 1.521687 & -1.176352 \\
$\mathrm{H} 35$ & -3.480632 & -1.711947 & 1.702696 \\
$\mathrm{H} 36$ & -1.873815 & -1.699227 & 2.465141 \\
$\mathrm{H} 37$ & -3.62289 & 0.675873 & 2.077049 \\
$\mathrm{H} 38$ & -1.996605 & 0.635376 & 2.798996 \\
$\mathrm{H} 39$ & -3.278672 & -2.678784 & -1.704928
\end{tabular}




\begin{tabular}{lrrr} 
H40 & -3.66414 & 2.583077 & -0.918918 \\
H41 & -4.177096 & -1.178456 & -2.350539 \\
H42 & -4.355208 & 1.224748 & -1.992216 \\
\hline
\end{tabular}

Table S10. The Cartesian Coordinates (in $\AA$ ), and Mülliken Spin $(\rho)$ and Charge $(q)$ Densities of $7^{\circ+}$ Obtained by UB3LYP/cc-pVDZ Calculation. (The total electronic energy is -850.108177314 Hartree.)

\begin{tabular}{|c|c|c|c|c|c|}
\hline & $X$ & $\mathrm{Y}$ & Z & $\rho$ & $q$ \\
\hline $\mathrm{C} 1$ & -0.906776 & -0.214587 & 0.357081 & 0.400337 & 0.119575 \\
\hline $\mathrm{C} 2$ & -1.729065 & -1.468049 & 0.156191 & -0.016947 & -0.056616 \\
\hline $\mathrm{C} 3$ & -2.029897 & 0.714761 & 0.762025 & -0.018177 & -0.063072 \\
\hline $\mathrm{C} 4$ & -2.718347 & -0.891355 & -0.874373 & 0.013013 & -0.052893 \\
\hline $\mathrm{C} 5$ & -2.952718 & 0.519452 & -0.447818 & 0.020831 & -0.041743 \\
\hline C6 & -2.476013 & -1.580677 & 1.518786 & 0.009646 & 0.075834 \\
\hline $\mathrm{C} 7$ & -2.638456 & -0.091218 & 1.958014 & 0.016291 & 0.084429 \\
\hline $\mathrm{C} 8$ & 0.461329 & 0.025455 & 0.15301 & 0.092739 & 0.047097 \\
\hline $\mathrm{C} 9$ & 1.398986 & -1.08692 & -0.024154 & 0.049359 & -0.031723 \\
\hline $\mathrm{C} 10$ & 0.992046 & 1.389881 & 0.108617 & 0.050522 & -0.037247 \\
\hline C11 & 2.460895 & -0.969237 & -0.962358 & 0.073014 & 0.040059 \\
\hline $\mathrm{C} 12$ & 2.257949 & 1.672687 & 0.692411 & 0.074784 & 0.038799 \\
\hline $\mathrm{C} 13$ & 3.355204 & -2.015949 & -1.156251 & -0.038734 & 0.057839 \\
\hline C14 & 2.779774 & 2.960715 & 0.664184 & -0.039458 & 0.05773 \\
\hline $\mathrm{C} 15$ & 3.235019 & -3.18918 & -0.399925 & 0.121811 & 0.064887 \\
\hline $\mathrm{C} 16$ & 2.07188 & 3.988501 & 0.027024 & 0.124376 & 0.065169 \\
\hline $\mathrm{C} 17$ & 2.206337 & -3.316056 & 0.548717 & -0.020394 & 0.05743 \\
\hline $\mathrm{C} 18$ & 0.830264 & 3.723943 & -0.575677 & -0.019688 & 0.055108 \\
\hline C19 & 1.291547 & -2.286907 & 0.72668 & 0.047946 & 0.038335 \\
\hline $\mathrm{C} 20$ & 0.287394 & 2.446947 & -0.525968 & 0.04714 & 0.03706 \\
\hline H21 & 2.541033 & -0.066185 & -1.56878 & -0.003002 & 0.00262 \\
\hline $\mathrm{H} 22$ & 2.799216 & 0.878133 & 1.207803 & -0.00308 & 0.001474 \\
\hline $\mathrm{H} 23$ & 4.146511 & -1.923861 & -1.902082 & 0.001836 & 0.014022 \\
\hline $\mathrm{H} 24$ & 3.737551 & 3.170539 & 1.14325 & 0.001844 & 0.01364 \\
\hline $\mathrm{H} 25$ & 3.941883 & -4.008207 & -0.547502 & -0.004871 & 0.020251 \\
\hline H26 & 2.485106 & 4.998832 & -0.001064 & -0.004972 & 0.020212 \\
\hline $\mathrm{H} 27$ & 2.129373 & -4.222624 & 1.151414 & 0.000621 & 0.012519 \\
\hline $\mathrm{H} 28$ & 0.293718 & 4.522869 & -1.090418 & 0.000603 & 0.012689 \\
\hline $\mathrm{H} 29$ & 0.517267 & -2.380481 & 1.488551 & -0.002472 & -0.01248 \\
\hline H30 & -0.660437 & 2.2436 & -1.025707 & -0.00253 & -0.002314 \\
\hline H31 & -1.216086 & -2.371275 & -0.184844 & 0.000523 & -0.012125 \\
\hline H32 & -1.78176 & 1.752109 & 1.000872 & 0.000329 & -0.014112 \\
\hline C33 & -3.212057 & -1.555611 & -1.927487 & 0.027547 & 0.055374 \\
\hline C34 & -3.762341 & 1.445912 & -0.974781 & -0.008482 & 0.047348 \\
\hline H35 & -3.434757 & -2.100965 & 1.391429 & 0.000867 & 0.040934 \\
\hline H36 & -1.880128 & -2.151876 & 2.246788 & -0.000353 & 0.029965 \\
\hline H37 & -3.683601 & 0.203902 & 2.120868 & 0.001856 & 0.041661 \\
\hline H38 & -2.078517 & 0.120749 & 2.880908 & -0.000154 & 0.028285 \\
\hline H39 & -2.916765 & -2.58485 & -2.140897 & -0.000015 & 0.0343 \\
\hline $\mathrm{H} 40$ & -3.81981 & 2.453653 & -0.557773 & 0.001464 & 0.030961 \\
\hline H41 & -3.934223 & -1.094913 & -2.605251 & 0.001285 & 0.040271 \\
\hline $\mathrm{H} 42$ & -4.403291 & 1.225791 & -1.831247 & 0.002744 & 0.038448 \\
\hline
\end{tabular}

Table S11. The Cartesian Coordinates (in $\AA$ ), and Mülliken Charge $(q)$ Densities of $7^{2+}$ Obtained by RB3LYP/cc-pVDZ Calculation. (The total electronic energy is -849.701512703 Hartree.) 


\begin{tabular}{|c|c|c|c|c|}
\hline & X & $\mathrm{Y}$ & Z & $q$ \\
\hline $\mathrm{C} 1$ & -0.952881 & -0.139232 & 0.307482 & 0.146151 \\
\hline $\mathrm{C} 2$ & -1.818267 & -1.378029 & 0.26777 & -0.05703 \\
\hline $\mathrm{C} 3$ & -2.010719 & 0.88033 & 0.605309 & -0.085306 \\
\hline $\mathrm{C} 4$ & -2.764693 & -0.84642 & -0.824749 & -0.065652 \\
\hline $\mathrm{C} 5$ & -3.037258 & 0.563925 & -0.477322 & -0.022519 \\
\hline C6 & -2.557496 & -1.310444 & 1.635033 & 0.080439 \\
\hline $\mathrm{C} 7$ & -2.579129 & 0.214751 & 1.950058 & 0.07685 \\
\hline $\mathrm{C} 8$ & 0.447762 & 0.017872 & 0.114314 & 0.070206 \\
\hline $\mathrm{C} 9$ & 1.320546 & -1.132909 & -0.040597 & -0.040877 \\
\hline $\mathrm{C} 10$ & 1.053929 & 1.337297 & 0.082899 & -0.042268 \\
\hline $\mathrm{C} 11$ & 2.411249 & -1.045786 & -0.971071 & 0.053863 \\
\hline $\mathrm{C} 12$ & 2.331425 & 1.523651 & 0.713791 & 0.054799 \\
\hline $\mathrm{C} 13$ & 3.288634 & -2.105566 & -1.134238 & 0.074207 \\
\hline $\mathrm{C} 14$ & 2.961631 & 2.756863 & 0.682482 & 0.075072 \\
\hline $\mathrm{C} 15$ & 3.124096 & -3.268853 & -0.363421 & 0.093212 \\
\hline $\mathrm{C} 16$ & 2.356576 & 3.829308 & 0.00508 & 0.09348 \\
\hline $\mathrm{C} 17$ & 2.070179 & -3.370215 & 0.574469 & 0.074074 \\
\hline C18 & 1.109283 & 3.664263 & -0.641863 & 0.074173 \\
\hline C19 & 1.169177 & -2.331874 & 0.722798 & 0.039899 \\
\hline $\mathrm{C} 20$ & 0.454012 & 2.448088 & -0.587874 & 0.038289 \\
\hline $\mathrm{H} 21$ & 2.50066 & -0.161123 & -1.603651 & 0.027474 \\
\hline $\mathrm{H} 22$ & 2.770012 & 0.701101 & 1.281095 & 0.028835 \\
\hline $\mathrm{H} 23$ & 4.092175 & -2.045491 & -1.869988 & 0.048773 \\
\hline $\mathrm{H} 24$ & 3.91306 & 2.901278 & 1.196782 & 0.049495 \\
\hline $\mathrm{H} 25$ & 3.811827 & -4.107902 & -0.492822 & 0.062088 \\
\hline $\mathrm{H} 26$ & 2.847832 & 4.805048 & -0.014712 & 0.062703 \\
\hline $\mathrm{H} 27$ & 1.978956 & -4.267054 & 1.18966 & 0.048098 \\
\hline $\mathrm{H} 28$ & 0.671141 & 4.499779 & -1.190467 & 0.047704 \\
\hline $\mathrm{H} 29$ & 0.393561 & -2.399624 & 1.485454 & 0.013541 \\
\hline $\mathrm{H} 30$ & -0.483526 & 2.317868 & -1.128617 & 0.017354 \\
\hline H31 & -1.351975 & -2.33246 & 0.012395 & 0.013463 \\
\hline H32 & -1.731295 & 1.92861 & 0.727334 & 0.016633 \\
\hline C33 & -3.215542 & -1.560635 & -1.887159 & 0.125089 \\
\hline C34 & -3.963694 & 1.402049 & -0.977302 & 0.104634 \\
\hline H35 & -3.560515 & -1.751581 & 1.56087 & 0.068206 \\
\hline H36 & -2.006291 & -1.871055 & 2.403879 & 0.060802 \\
\hline H37 & -3.5772 & 0.622475 & 2.151681 & 0.076865 \\
\hline H38 & -1.920322 & 0.480185 & 2.78797 & 0.060844 \\
\hline H39 & -2.819009 & -2.552335 & -2.118436 & 0.08726 \\
\hline $\mathrm{H} 40$ & -4.102558 & 2.406054 & -0.56908 & 0.076286 \\
\hline H41 & -4.002836 & -1.175606 & -2.541044 & 0.094342 \\
\hline $\mathrm{H} 42$ & -4.616615 & 1.111218 & -1.80364 & 0.078452 \\
\hline
\end{tabular}

Table S12. The Cartesian Coordinates (in $\AA$ ), and Mülliken Spin $(\rho)$ and Charge $(q)$ Densities of $7^{--}$Obtained by UB3LYP/cc-pVDZ Calculation. (The total electronic energy is -850.372266901 Hartree.)

\begin{tabular}{rrrrrr}
\hline & $\mathrm{X}$ & \multicolumn{1}{c}{$\mathrm{Y}$} & \multicolumn{1}{c}{$\mathrm{Z}$} & \multicolumn{1}{c}{$\rho$} & \multicolumn{1}{c}{$q$} \\
\hline $\mathrm{C} 1$ & -0.859776 & -0.306071 & 0.362584 & 0.251837 & 0.005506 \\
$\mathrm{C} 2$ & -1.607747 & -1.610673 & 0.110905 & -0.022199 & -0.037121 \\
$\mathrm{C} 3$ & -2.076264 & 0.483315 & 0.825149 & -0.019027 & -0.042166 \\
$\mathrm{C} 4$ & -2.620431 & -1.064107 & -0.896357 & -0.0032 & -0.01905 \\
$\mathrm{C} 5$ & -2.947833 & 0.292164 & -0.416146 & 0.012208 & -0.01753 \\
C6 & -2.33793 & -1.862125 & 1.465699 & 0.013127 & 0.08904
\end{tabular}




\begin{tabular}{lrrrrr} 
C7 & -2.640903 & -0.416781 & 1.96929 & 0.016712 & 0.094924 \\
C8 & 0.46999 & 0.055177 & 0.149632 & 0.077645 & -0.029754 \\
C9 & 1.512102 & -0.976053 & -0.013582 & 0.023264 & -0.022686 \\
C10 & 0.871302 & 1.472474 & 0.088551 & 0.024502 & -0.032616 \\
C11 & 2.592171 & -0.812796 & -0.924062 & 0.045792 & 0.006454 \\
C12 & 2.10971 & 1.923646 & 0.621987 & 0.038088 & 0.003933 \\
C13 & 3.563096 & -1.79528 & -1.100346 & -0.019017 & 0.034976 \\
C14 & 2.474778 & 3.266908 & 0.60355 & -0.014265 & 0.032859 \\
C15 & 3.509274 & -2.999972 & -0.381232 & 0.093522 & -0.000164 \\
C16 & 1.619077 & 4.237273 & 0.056351 & 0.089342 & 0.000143 \\
C17 & 2.457572 & -3.183853 & 0.528428 & -0.021979 & 0.035562 \\
C18 & 0.393374 & 3.817461 & -0.47805 & -0.024825 & 0.0315 \\
C19 & 1.487869 & -2.199456 & 0.709524 & 0.048604 & 0.013101 \\
C20 & 0.025983 & 2.471774 & -0.467312 & 0.053765 & 0.005664 \\
H21 & 2.646517 & 0.105989 & -1.511947 & -0.002243 & -0.05505 \\
H22 & 2.784981 & 1.19178 & 1.070898 & -0.002059 & -0.059269 \\
H23 & 4.370075 & -1.626779 & -1.821098 & 0.000531 & -0.068958 \\
H24 & 3.436453 & 3.56712 & 1.032846 & 0.000315 & -0.07151 \\
H25 & 4.268054 & -3.773676 & -0.525434 & -0.004653 & -0.078109 \\
H26 & 1.903741 & 5.292907 & 0.045429 & -0.004518 & -0.079025 \\
H27 & 2.401664 & -4.105003 & 1.118038 & 0.001395 & -0.070998 \\
H28 & -0.284955 & 4.550845 & -0.92636 & 0.001069 & -0.066923 \\
H29 & 0.701861 & -2.351369 & 1.451526 & -0.001788 & -0.065747 \\
H30 & -0.917057 & 2.17004 & -0.925654 & -0.00335 & -0.038408 \\
H31 & -1.036379 & -2.465951 & -0.266127 & -0.000039 & -0.066826 \\
H32 & -1.928731 & 1.529429 & 1.11689 & 0.001877 & -0.072477 \\
C33 & -3.044423 & -1.693701 & -2.022084 & 0.206477 & -0.045639 \\
C34 & -3.775845 & 1.218084 & -0.96122 & 0.156167 & -0.043236 \\
H35 & -3.251475 & -2.464452 & 1.32993 & 0.002453 & -0.030145 \\
H36 & -1.679284 & -2.40007 & 2.169425 & 0.003841 & -0.028099 \\
H37 & -3.717201 & -0.239933 & 2.132974 & 0.004481 & -0.030667 \\
H38 & -2.111061 & -0.20548 & 2.913536 & 0.00288 & -0.030202 \\
H39 & -2.728438 & -2.714638 & -2.251186 & -0.008335 & -0.040669 \\
H40 & -3.929193 & 2.188516 & -0.481994 & -0.006453 & -0.037712 \\
H41 & -3.680628 & -1.190408 & -2.755547 & -0.006458 & -0.037508 \\
H42 & -4.299827 & 1.036689 & -1.903705 & -0.005486 & -0.035397 \\
\hline & & & & & \\
\hline
\end{tabular}

Table S13. The Cartesian Coordinates (in $\AA$ ) of $\mathbf{8}$ Optimized by RB3LYP/cc-pVDZ. (The total electronic energy is -849.131277964 Hartree.)

\begin{tabular}{rrrr}
\hline & \multicolumn{1}{c}{$\mathrm{X}$} & \multicolumn{1}{c}{$\mathrm{Y}$} & \multicolumn{1}{c}{$\mathrm{Z}$} \\
\hline $\mathrm{C} 1$ & -0.906635 & -0.147705 & 0.390155 \\
$\mathrm{C} 2$ & -1.804453 & -1.387172 & 0.377905 \\
$\mathrm{C} 3$ & -2.022748 & 0.846281 & 0.721765 \\
$\mathrm{C} 4$ & -2.828173 & -0.924617 & -0.680904 \\
$\mathrm{C} 5$ & -2.974835 & 0.542763 & -0.452015 \\
$\mathrm{C} 6$ & -2.509081 & -1.196019 & 1.726349 \\
$\mathrm{C} 7$ & -2.63603 & 0.12564 & 1.930408 \\
$\mathrm{C} 8$ & 0.410763 & 0.016258 & 0.161003 \\
$\mathrm{C} 9$ & 1.318601 & -1.156144 & -0.032024 \\
$\mathrm{C} 10$ & 1.031052 & 1.375154 & 0.095425 \\
$\mathrm{C} 11$ & 2.208276 & -1.195903 & -1.122111 \\
$\mathrm{C} 12$ & 2.208175 & 1.656201 & 0.814397 \\
$\mathrm{C} 13$ & 3.052205 & -2.290097 & -1.318487
\end{tabular}




\begin{tabular}{lrrr} 
C14 & 2.791162 & 2.923643 & 0.767991 \\
C15 & 3.037808 & -3.362376 & -0.419976 \\
C16 & 2.218834 & 3.934955 & -0.010917 \\
C17 & 2.173457 & -3.328385 & 0.677538 \\
C18 & 1.060648 & 3.665148 & -0.744991 \\
C19 & 1.323792 & -2.235094 & 0.870147 \\
C20 & 0.473597 & 2.397534 & -0.694234 \\
H21 & 2.228969 & -0.360874 & -1.825682 \\
H22 & 2.664159 & 0.8707 & 1.420857 \\
H23 & 3.726645 & -2.305674 & -2.178062 \\
H24 & 3.699077 & 3.122844 & 1.342467 \\
H25 & 3.702681 & -4.215914 & -0.570974 \\
H26 & 2.678543 & 4.925129 & -0.051604 \\
H27 & 2.165314 & -4.153031 & 1.394459 \\
H28 & 0.614577 & 4.442659 & -1.369968 \\
H29 & 0.667972 & -2.201536 & 1.74268 \\
H30 & -0.417044 & 2.186757 & -1.289643 \\
H31 & -1.351838 & -2.363041 & 0.182157 \\
H32 & -1.766359 & 1.900564 & 0.856963 \\
C33 & -3.415939 & -1.696421 & -1.602582 \\
C34 & -3.728748 & 1.430059 & -1.112663 \\
H35 & -2.914778 & -2.006939 & 2.332151 \\
H36 & -3.165405 & 0.629128 & 2.739975 \\
H37 & -3.193101 & -2.763414 & -1.673299 \\
H38 & -3.721683 & 2.489196 & -0.845018 \\
H39 & -4.133677 & -1.288543 & -2.318798 \\
H40 & -4.374411 & 1.129783 & -1.941738 \\
\hline & & &
\end{tabular}

Table S14. The Cartesian Coordinates (in $\AA$ ), and Mülliken Spin $(\rho)$ and Charge $(q)$ Densities of $\mathbf{8}^{\boldsymbol{0}^{+}}$Obtained by UB3LYP/cc-pVDZ Calculation. (The total electronic energy is -848.871058430 Hartree.)

\begin{tabular}{lrrrrr}
\hline & \multicolumn{1}{c}{$\mathrm{X}$} & \multicolumn{1}{c}{$\mathrm{Y}$} & \multicolumn{1}{c}{$\mathrm{Z}$} & \multicolumn{1}{c}{$\rho$} & \multicolumn{1}{c}{$q$} \\
\hline $\mathrm{C} 1$ & -0.91537 & -0.219446 & 0.283132 & 0.34115 & 0.119976 \\
$\mathrm{C} 2$ & -1.740508 & -1.488817 & 0.126499 & -0.012927 & -0.018434 \\
$\mathrm{C} 3$ & -2.041757 & 0.680791 & 0.772538 & -0.016477 & -0.02665 \\
$\mathrm{C} 4$ & -2.901998 & -0.96762 & -0.737885 & 0.000666 & -0.036938 \\
$\mathrm{C} 5$ & -3.104963 & 0.442242 & -0.310152 & -0.001289 & -0.030867 \\
$\mathrm{C} 6$ & -2.214796 & -1.504248 & 1.584923 & 0.070503 & 0.076999 \\
$\mathrm{C} 7$ & -2.388642 & -0.217701 & 1.9682 & 0.07146 & 0.090045 \\
$\mathrm{C} 8$ & 0.453824 & 0.02719 & 0.115965 & 0.131497 & 0.056529 \\
$\mathrm{C} 9$ & 1.405516 & -1.076828 & -0.045196 & 0.029815 & -0.038119 \\
$\mathrm{C} 10$ & 0.958848 & 1.402323 & 0.069454 & 0.038014 & -0.035399 \\
$\mathrm{C} 11$ & 2.467283 & -0.959334 & -0.980864 & 0.073233 & 0.037909 \\
$\mathrm{C} 12$ & 2.217764 & 1.711015 & 0.651492 & 0.070588 & 0.037101 \\
$\mathrm{C} 13$ & 3.370571 & -2.001739 & -1.161929 & -0.037961 & 0.057119 \\
$\mathrm{C} 14$ & 2.709446 & 3.011324 & 0.627041 & -0.036725 & 0.057103 \\
$\mathrm{C} 15$ & 3.256881 & -3.168332 & -0.395723 & 0.108351 & 0.06231 \\
$\mathrm{C} 16$ & 1.978181 & 4.02584 & -0.004871 & 0.11649 & 0.063525 \\
$\mathrm{C} 17$ & 2.227289 & -3.292643 & 0.551279 & -0.017121 & 0.056497 \\
$\mathrm{C} 18$ & 0.743475 & 3.735391 & -0.607278 & -0.024676 & 0.056565 \\
$\mathrm{C} 19$ & 1.304727 & -2.267113 & 0.717524 & 0.044039 & 0.034227 \\
$\mathrm{C} 20$ & 0.23157 & 2.444761 & -0.563192 & 0.052793 & 0.028214 \\
$\mathrm{H} 21$ & 2.543967 & -0.060517 & -1.594142 & -0.003025 & 0.000666 \\
$\mathrm{H} 22$ & 2.779176 & 0.926941 & 1.161098 & -0.002918 & 0.00111 \\
& & & & &
\end{tabular}




\begin{tabular}{rrrrrr} 
H23 & 4.164411 & -1.909479 & -1.905106 & 0.001905 & 0.012422 \\
H24 & 3.66346 & 3.2405 & 1.104869 & 0.00189 & 0.012683 \\
H25 & 3.971004 & -3.982895 & -0.532407 & -0.004391 & 0.017849 \\
H26 & 2.369567 & 5.044844 & -0.030863 & -0.004702 & 0.018422 \\
H27 & 2.156123 & -4.19304 & 1.163994 & 0.000586 & 0.010484 \\
H28 & 0.18809 & 4.522942 & -1.119567 & 0.000797 & 0.011428 \\
H29 & 0.530586 & -2.354972 & 1.48095 & -0.003232 & -0.0164 \\
H30 & -0.709916 & 2.22076 & -1.06673 & -0.002855 & -0.001921 \\
H31 & -1.249031 & -2.392039 & -0.242383 & 0.000502 & -0.008874 \\
H32 & -1.818615 & 1.722354 & 1.015002 & 0.000931 & -0.009728 \\
C33 & -3.544912 & -1.676228 & -1.673519 & 0.004107 & 0.062578 \\
C34 & -3.987322 & 1.350899 & -0.744593 & 0.0142 & 0.055501 \\
H35 & -2.395851 & -2.405493 & 2.17165 & -0.001835 & 0.021871 \\
H36 & -2.735974 & 0.152524 & 2.933075 & -0.002277 & 0.022421 \\
H37 & -3.265552 & -2.707472 & -1.89844 & -0.000131 & 0.03228 \\
H38 & -4.005644 & 2.364748 & -0.3394 & -0.000461 & 0.030633 \\
H39 & -4.37211 & -1.249767 & -2.245062 & -0.000152 & 0.03883 \\
H40 & -4.724332 & 1.109692 & -1.513617 & -0.000364 & 0.040036 \\
\hline
\end{tabular}

Table S15. The Cartesian Coordinates (in $\AA$ ), and Mülliken Charge $(q)$ Densities of $\mathbf{8}^{2+}$ Obtained by RB3LYP/cc-pVDZ Calculation. (The total electronic energy is -848.485174404 Hartree.)

\begin{tabular}{rrrrr}
\hline & \multicolumn{1}{c}{$\mathrm{X}$} & \multicolumn{1}{c}{$\mathrm{Y}$} & \multicolumn{1}{c}{$\mathrm{Z}$} & \multicolumn{1}{c}{$q$} \\
\hline $\mathrm{C} 1$ & 0.718256 & -0.717434 & -0.224745 & -0.120197 \\
$\mathrm{C} 2$ & 1.415132 & -1.490395 & 0.954686 & 0.060084 \\
$\mathrm{C} 3$ & 1.896385 & -0.684318 & -1.22417 & 0.049267 \\
$\mathrm{C} 4$ & 2.879516 & -1.144436 & 0.918262 & -0.057143 \\
$\mathrm{C} 5$ & 3.190425 & -0.672587 & -0.44669 & -0.065454 \\
$\mathrm{C} 6$ & 0.886855 & -2.454827 & -0.048016 & 0.15517 \\
$\mathrm{C} 7$ & 1.180377 & -1.992386 & -1.340305 & 0.148383 \\
$\mathrm{C} 8$ & -0.540322 & 0.071596 & -0.14994 & 0.139228 \\
$\mathrm{C} 9$ & -1.834098 & -0.557356 & -0.029221 & -0.026857 \\
$\mathrm{C} 10$ & -0.372183 & 1.490348 & -0.101018 & 0.00023 \\
$\mathrm{C} 11$ & -2.794782 & 0.006567 & 0.867798 & 0.024412 \\
$\mathrm{C} 12$ & -1.457454 & 2.353115 & -0.475561 & 0.029646 \\
$\mathrm{C} 13$ & -4.042323 & -0.581809 & 1.032524 & 0.073373 \\
$\mathrm{C} 14$ & -1.309824 & 3.729656 & -0.434192 & 0.074652 \\
$\mathrm{C} 15$ & -4.383901 & -1.719872 & 0.290177 & 0.086013 \\
$\mathrm{C} 16$ & -0.092378 & 4.291567 & -0.016135 & 0.09675 \\
$\mathrm{C} 17$ & -3.462052 & -2.282565 & -0.61282 & 0.070395 \\
$\mathrm{C} 18$ & 0.993914 & 3.468854 & 0.344471 & 0.074191 \\
$\mathrm{C} 19$ & -2.199417 & -1.729713 & -0.755257 & 0.004446 \\
$\mathrm{C} 20$ & 0.870269 & 2.093271 & 0.286071 & 0.042838 \\
$\mathrm{H} 21$ & -2.515589 & 0.859994 & 1.48651 & 0.026978 \\
$\mathrm{H} 22$ & -2.379677 & 1.920719 & -0.863022 & 0.036594 \\
$\mathrm{H} 23$ & -4.750119 & -0.161151 & 1.748804 & 0.046093 \\
$\mathrm{H} 24$ & -2.131273 & 4.376043 & -0.74733 & 0.048514 \\
$\mathrm{H} 25$ & -5.369035 & -2.175403 & 0.412425 & 0.055559 \\
$\mathrm{H} 26$ & 0.020418 & 5.377864 & 0.014972 & 0.059314 \\
$\mathrm{H} 27$ & -3.747849 & -3.149006 & -1.211677 & 0.041077 \\
$\mathrm{H} 28$ & 1.931172 & 3.920261 & 0.673309 & 0.041852 \\
$\mathrm{H} 29$ & -1.52781 & -2.15063 & -1.50253 & -0.017474 \\
$\mathrm{H} 30$ & 1.713172 & 1.47438 & 0.595432 & 0.013877 \\
$\mathrm{H} 31$ & 0.905053 & -1.528632 & 1.920263 & 0.045145
\end{tabular}




\begin{tabular}{rrrrr} 
H32 & 1.805453 & -0.047651 & -2.107403 & 0.043912 \\
C33 & 3.678298 & -1.255483 & 1.990938 & 0.112992 \\
C34 & 4.360398 & -0.276766 & -0.96864 & 0.110512 \\
H35 & 0.115651 & -3.197312 & 0.169591 & 0.09612 \\
H36 & 0.680497 & -2.347655 & -2.24581 & 0.105973 \\
H37 & 3.300954 & -1.603178 & 2.95457 & 0.064293 \\
H38 & 4.440706 & 0.065318 & -2.002287 & 0.062478 \\
H39 & 4.741794 & -1.013892 & 1.934838 & 0.074309 \\
H40 & 5.279941 & -0.290236 & -0.379964 & 0.072458 \\
\hline
\end{tabular}

Table S16. The Cartesian Coordinates (in $\AA$ ), and Mülliken Spin $(\rho)$ and Charge $(q)$ Densities of $\mathbf{8}^{*-}$ Obtained by UB3LYP/cc-pVDZ Calculation. (The total electronic energy is -849.133273009 Hartree.)

\begin{tabular}{|c|c|c|c|c|c|}
\hline & $\mathrm{X}$ & $\mathrm{Y}$ & $\mathrm{Z}$ & $\rho$ & $q$ \\
\hline $\mathrm{C} 1$ & -0.855289 & -0.372156 & 0.371206 & 0.263847 & 0.036821 \\
\hline $\mathrm{C} 2$ & -1.527351 & -1.726743 & 0.143143 & -0.022041 & -0.013728 \\
\hline $\mathrm{C} 3$ & -2.108087 & 0.310225 & 0.919439 & -0.017408 & -0.014337 \\
\hline $\mathrm{C} 4$ & -2.601157 & -1.222483 & -0.841696 & -0.007602 & -0.009981 \\
\hline $\mathrm{C} 5$ & -2.998522 & 0.098646 & -0.325467 & 0.016075 & -0.009399 \\
\hline C6 & -2.225994 & -1.937623 & 1.492999 & 0.015507 & 0.050485 \\
\hline $\mathrm{C} 7$ & -2.56098 & -0.722319 & 1.960363 & 0.013631 & 0.051187 \\
\hline $\mathrm{C} 8$ & 0.446851 & 0.071659 & 0.151797 & 0.073673 & -0.022254 \\
\hline C9 & 1.544651 & -0.899126 & -0.007209 & 0.023302 & -0.030063 \\
\hline $\mathrm{C} 10$ & 0.751022 & 1.510872 & 0.085589 & 0.024086 & -0.030029 \\
\hline $\mathrm{C} 11$ & 2.609418 & -0.688968 & -0.926031 & 0.047687 & 0.004623 \\
\hline $\mathrm{C} 12$ & 1.971202 & 2.045509 & 0.583288 & 0.039483 & 0.001193 \\
\hline $\mathrm{C} 13$ & 3.630984 & -1.620246 & -1.094404 & -0.020334 & 0.035119 \\
\hline C14 & 2.24165 & 3.410876 & 0.557631 & -0.015468 & 0.033195 \\
\hline $\mathrm{C} 15$ & 3.644538 & -2.816915 & -0.360148 & 0.094773 & -0.000884 \\
\hline $\mathrm{C} 16$ & 1.30496 & 4.321004 & 0.040563 & 0.090283 & -0.000286 \\
\hline $\mathrm{C} 17$ & 2.607754 & -3.046291 & 0.556619 & -0.021954 & 0.036687 \\
\hline $\mathrm{C} 18$ & 0.09434 & 3.818378 & -0.456074 & -0.02516 & 0.031896 \\
\hline C19 & 1.587862 & -2.11282 & 0.731697 & 0.048304 & 0.007887 \\
\hline $\mathrm{C} 20$ & -0.178702 & 2.450891 & -0.439152 & 0.054622 & 0.003267 \\
\hline $\mathrm{H} 21$ & 2.611438 & 0.224264 & -1.525157 & -0.002352 & -0.055887 \\
\hline $\mathrm{H} 22$ & 2.708521 & 1.36183 & 1.00989 & -0.002124 & -0.058633 \\
\hline $\mathrm{H} 23$ & 4.425222 & -1.418048 & -1.820671 & 0.000579 & -0.069277 \\
\hline $\mathrm{H} 24$ & 3.193331 & 3.776076 & 0.95799 & 0.000363 & -0.071706 \\
\hline $\mathrm{H} 25$ & 4.443764 & -3.550021 & -0.497978 & -0.004751 & -0.078404 \\
\hline $\mathrm{H} 26$ & 1.516191 & 5.393658 & 0.024203 & -0.004554 & -0.079596 \\
\hline $\mathrm{H} 27$ & 2.604221 & -3.962057 & 1.157202 & 0.001233 & -0.070151 \\
\hline $\mathrm{H} 28$ & -0.647003 & 4.503883 & -0.879801 & 0.001083 & -0.067482 \\
\hline $\mathrm{H} 29$ & 0.812524 & -2.294828 & 1.478684 & -0.00233 & -0.056203 \\
\hline $\mathrm{H} 30$ & -1.113165 & 2.084923 & -0.86769 & -0.003422 & -0.038977 \\
\hline H31 & -0.925449 & -2.557169 & -0.239042 & 0.000103 & -0.068576 \\
\hline H32 & -2.035287 & 1.348058 & 1.261483 & 0.001048 & -0.072146 \\
\hline $\mathrm{C} 33$ & -3.014848 & -1.865105 & -1.963204 & 0.218367 & -0.044533 \\
\hline C34 & -3.884969 & 0.992224 & -0.825656 & 0.143818 & -0.041478 \\
\hline H35 & -2.486862 & -2.907992 & 1.922383 & 0.001747 & -0.068995 \\
\hline H36 & -3.146406 & -0.493492 & 2.854309 & 0.002765 & -0.070474 \\
\hline H37 & -2.634873 & -2.857793 & -2.217702 & -0.008829 & -0.040649 \\
\hline H38 & -4.081223 & 1.938589 & -0.315134 & -0.005834 & -0.036807 \\
\hline H39 & -3.708665 & -1.398218 & -2.668042 & -0.007436 & -0.037311 \\
\hline $\mathrm{H} 40$ & -4.412274 & 0.811409 & -1.766689 & -0.00478 & -0.034115 \\
\hline
\end{tabular}


Table S17. The Cartesian Coordinates (in $\AA$ ), and Mülliken Charge $(q)$ Densities of 1' Obtained by RB3LYP/aug-cc-pVDZ Calculation. (The total electronic energy is -272.087116458 Hartree.)

\begin{tabular}{rrrrr}
\hline & \multicolumn{1}{c}{$\mathrm{X}$} & \multicolumn{1}{c}{$\mathrm{Y}$} & $\mathrm{Z}$ & $\mathrm{q}$ \\
\hline $\mathrm{C} 1$ & 0.00111 & 0.000011 & 1.458321 & 0.133082 \\
$\mathrm{C} 2$ & 0.087086 & 1.113092 & 0.383184 & 0.837541 \\
$\mathrm{C} 3$ & 0.087017 & -1.113092 & 0.383199 & 0.837534 \\
C4 & 1.252301 & 0.674292 & -0.524751 & 0.597591 \\
C5 & 1.252239 & -0.674372 & -0.524767 & 0.597601 \\
C6 & -1.204979 & 0.781059 & -0.451375 & 1.064275 \\
C7 & -1.205036 & -0.780997 & -0.451351 & 1.064285 \\
H8 & 0.936241 & -0.000013 & 2.058074 & -0.633334 \\
H9 & 0.128178 & 2.157963 & 0.726813 & -0.811634 \\
H10 & 0.128053 & -2.15796 & 0.726842 & -0.81163 \\
H11 & 1.863411 & 1.318314 & -1.169288 & -0.778573 \\
H12 & 1.863287 & -1.318434 & -1.169323 & -0.778561 \\
H13 & -2.087124 & 1.177583 & 0.068663 & -0.616082 \\
H14 & -2.087202 & -1.177445 & 0.068708 & -0.616098 \\
H15 & -1.181685 & -1.199389 & -1.472605 & -0.542997 \\
H16 & -1.181589 & 1.19942 & -1.472641 & -0.543001 \\
\hline
\end{tabular}

Table S18. The Cartesian Coordinates (in $\AA$ ), and Charge $(q)$ Densities of $1^{\circ}$ Obtained by UB3LYP/cc-pVDZ Calculation. (The total electronic energy is -272.066374222 Hartree.)

\begin{tabular}{rrrrr}
\hline & \multicolumn{1}{c}{$\mathrm{X}$} & \multicolumn{1}{c}{$\mathrm{Y}$} & \multicolumn{1}{c}{$\mathrm{Z}$} & \multicolumn{1}{c}{$\rho$} \\
\hline $\mathrm{C} 1$ & 0.143245 & 0 & 1.367443 & 0.970348 \\
$\mathrm{C} 2$ & 0.087768 & 1.133277 & 0.356089 & -0.039438 \\
C3 & 0.087764 & -1.133277 & 0.35609 & -0.039438 \\
C4 & 1.236287 & 0.673606 & -0.548996 & 0.054058 \\
C5 & 1.236283 & -0.67361 & -0.548997 & 0.054058 \\
C6 & -1.240225 & 0.780024 & -0.39814 & 0.004822 \\
C7 & -1.240228 & -0.78002 & -0.398138 & 0.004822 \\
H8 & 0.819695 & 0 & 2.228592 & -0.023044 \\
H9 & 0.148592 & 2.164767 & 0.725191 & 0.00205 \\
H10 & 0.148584 & -2.164767 & 0.725193 & 0.00205 \\
H11 & 1.874426 & 1.333936 & -1.140335 & 0.002426 \\
H12 & 1.874418 & -1.333943 & -1.140338 & 0.002426 \\
H13 & -2.104933 & 1.177369 & 0.155784 & 0.000384 \\
H14 & -2.104938 & -1.17736 & 0.155787 & 0.000384 \\
H15 & -1.260607 & -1.210008 & -1.410989 & 0.002046 \\
H16 & -1.260602 & 1.21001 & -1.410991 & 0.002046 \\
\hline
\end{tabular}

Table S19. The Cartesian Coordinates (in $\AA$ ), and Mülliken Charge $(q)$ Densities of $\mathbf{1}^{+}$Obtained by RB3LYP/cc-pVDZ Calculation. (The total electronic energy is -271.843409938 Hartree.)

\begin{tabular}{rrrrr}
\hline & $\mathrm{X}$ & \multicolumn{1}{c}{$\mathrm{Y}$} & \multicolumn{1}{c}{$\mathrm{Z}$} & \multicolumn{1}{c}{$\mathrm{q}$} \\
\hline $\mathrm{C} 1$ & -0.667715 & 0 & 0.966384 & 0.069149 \\
$\mathrm{C} 2$ & -0.023366 & -1.184312 & 0.250829 & -0.036785 \\
$\mathrm{C} 3$ & -0.023366 & 1.184312 & 0.250829 & -0.036785 \\
C4 & -1.170204 & -0.700534 & -0.570022 & 0.116084 \\
C5 & -1.170204 & 0.700534 & -0.570022 & 0.116084 \\
C6 & 1.391293 & -0.773367 & -0.185512 & 0.068291
\end{tabular}




\begin{tabular}{crrrr} 
C7 & 1.391293 & 0.773367 & -0.185512 & 0.068291 \\
H8 & -1.381267 & 0 & 1.790731 & 0.091083 \\
H9 & -0.170722 & -2.18384 & 0.667342 & 0.053033 \\
H10 & -0.170722 & 2.18384 & 0.667342 & 0.053033 \\
H11 & -2.060798 & -1.293765 & -0.795477 & 0.085595 \\
H12 & -2.060798 & 1.293765 & -0.795477 & 0.085595 \\
H13 & 2.11724 & -1.180644 & 0.531936 & 0.067868 \\
H14 & 2.11724 & 1.180644 & 0.531936 & 0.067868 \\
H15 & 1.621722 & 1.203311 & -1.170087 & 0.065798 \\
H16 & 1.621722 & -1.203311 & -1.170087 & 0.065798 \\
\hline
\end{tabular}

(4) The definition of angles.

According to the example of Laube (ref. 8), angles are defined as follows.

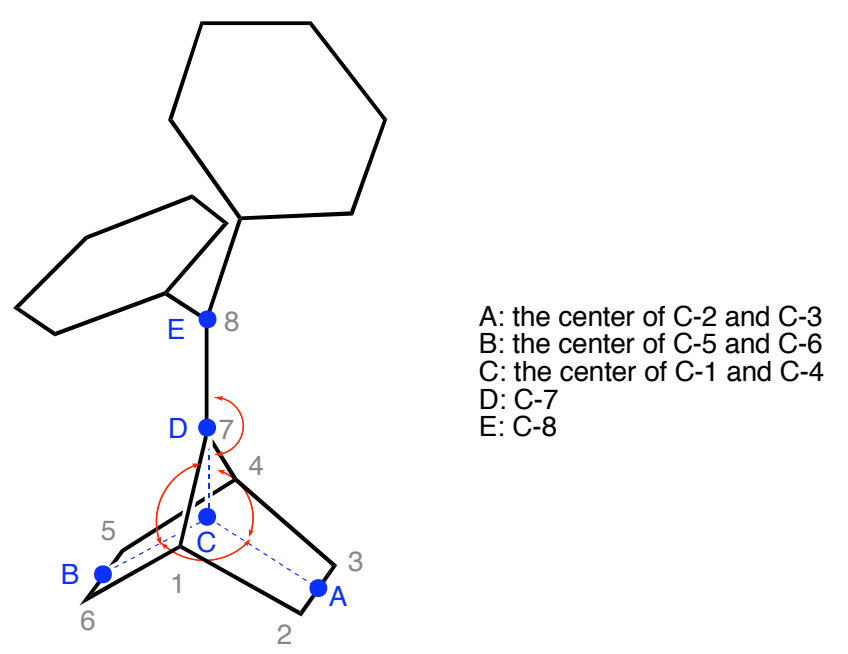

(end) 